\title{
Modulation Spectra Morphological Parameters: A New Method to Assess Voice Pathologies according to the GRBAS Scale
}

\author{
Laureano Moro-Velázquez, Jorge Andrés Gómez-García, \\ Juan Ignacio Godino-Llorente, and Gustavo Andrade-Miranda \\ ETSIST, Universidad Politécnica de Madrid, Campus Sur, Carretera de Valencia km 7, 28031 Madrid, Spain \\ Correspondence should be addressed to Laureano Moro-Velázquez; laureano.moro@upm.es
}

Received 23 January 2015; Revised 4 May 2015; Accepted 4 May 2015

Academic Editor: Adam Klein

Copyright ( 2015 Laureano Moro-Velázquez et al. This is an open access article distributed under the Creative Commons Attribution License, which permits unrestricted use, distribution, and reproduction in any medium, provided the original work is properly cited.

\begin{abstract}
Disordered voices are frequently assessed by speech pathologists using perceptual evaluations. This might lead to problems caused by the subjective nature of the process and due to the influence of external factors which compromise the quality of the assessment. In order to increase the reliability of the evaluations, the design of automatic evaluation systems is desirable. With that in mind, this paper presents an automatic system which assesses the Grade and Roughness level of the speech according to the GRBAS perceptual scale. Two parameterization methods are used: one based on the classic Mel-Frequency Cepstral Coefficients, which has already been used successfully in previous works, and other derived from modulation spectra. For the latter, a new group of parameters has been proposed, named Modulation Spectra Morphological Parameters: MSC, DRB, LMR, MSH, MSW, CIL, PALA, and RALA. In methodology, PCA and LDA are employed to reduce the dimensionality of feature space, and GMM classifiers to evaluate the ability of the proposed features on distinguishing the different levels. Efficiencies of $81.6 \%$ and $84.7 \%$ are obtained for Grade and Roughness, respectively, using modulation spectra parameters, while MFCCs performed $80.5 \%$ and $77.7 \%$. The obtained results suggest the usefulness of the proposed Modulation Spectra Morphological Parameters for automatic evaluation of Grade and Roughness in the speech.
\end{abstract}

\section{Introduction}

With the aim of diagnosing and evaluating the presence of a voice disorder clinicians and specialists have developed different assessment procedures [1] such as exploration using laryngoscopic techniques, acoustic analysis, or perceptual evaluations. The latter is widely used by clinicians to quantify the extent of a dysphony. Some well-known perceptual evaluation procedures are the Buffalo Rating Voice Profile [2], Consensus Auditory Perceptual Evaluation of Voice (CAPEV) [3], and GRBAS [4]. The main problem with perceptual analysis is the high intra/interrater variability $[5,6]$ due to the subjectivity of the assessment in which the experience of the evaluator, his/her physical fatigue, mental condition, and some other factors are involved. Hence, means such as acoustic analysis based on signal processing might be valuable in clinical scenarios, providing objective tools and indices which can directly represent the level of affection or at least help clinicians to make a more reliable and less subjective perceptual assessment. This noninvasive technique can complement and even replace other invasive methods of evaluation.

Besides, the large amount of improvements in the field of speech signal processing is addressed mostly to areas such as speech or speaker recognition. Many of these advances are being transferred to biomedical applications for clinical purposes; some recent examples are related to different uses such as telemonitoring of patients [7], telerehabilitation [8], or clinical-support systems [9]. However, there is a substantial quantity of research to be done for further enhancements. Roughly speaking, most of the studies in this field can be divided into three main categories: the first one is focused on developing automatic detectors of pathological voices 
[10-14] capable of categorizing voices between normal and pathological; the second group works with classifiers of pathologies $[11,15,16]$ which consists in determining the speech disorder of the speaker using the acoustic material; and the third and last group aims to evaluate and assess the voice quality $[8,17-23]$. The present study can be framed in the third mentioned category, highlighting the fact that the main goal is the development of new parameterization methods.

The essential common characteristic of all the automatic systems found in the literature is the need to extract a set of parameters from the acoustic signal to accomplish a further classification task. Regarding these parameters, some works use amplitude perturbations such as Shimmer or Amplitude Tremor Intensity Index (ATRI) [24-26] as input features while others are centered on frequency perturbations using Jitter $[8,25,26]$, frequency and cepstral-based analysis $[8$, $13,14,16,17,27,28], F_{0}$ Tremor Intensity Index $\left(F_{0}\right.$ TRI) $[24,26]$, or Linear Predictive Coding (LPC) [29]. Noisebased parameters $[30,31]$ and nonlinear analysis features $[12,18,25,32]$ are likewise widely used in this kind of automatic detectors. Moreover, other varieties of featureextraction techniques such as biomechanical attributes or signatures can be applied for the same purposes [10].

Focusing on the third kind of the aforementioned categories of detectors, those assessing the quality of voice, some are employed for simulating a perceptual evaluation such as GRBAS. For instance, several classification methods were used in $[19,20]$ to study the influence of the voice signal bandwidth in perceptual ratings and automatic evaluation of GRBAS Grade $(G)$ trait using cepstral parameters (Linear Frequency Spectrum Coefficients and Mel-Frequency Spectrum Coefficients). Efficiencies up to $80 \%$ were obtained using Gaussian Mixture Models (GMM) classifiers and leavex-out [33] cross-validation techniques. Similar parameterization methods were used in [9] to automatically evaluate $G$ with a Back-and-Forth Methodology in which there is feedback between the human experts that rated the database and the automatic detector, and vice versa. On [22] a group of 92 features comprising different types of measurements such as noise, cepstral and frequency parameters among others were used to detect GRBAS Breathiness $(B)$. After a reduction to a four-dimensional space, a $77 \%$ of efficiency was achieved using a 10-fold cross-validation scheme. Authors in [34] fulfilled a statistical study of acoustic measures provided by two commonly used analysis systems, Multidimensional Voice Analysis Program by Kay Elemetrics and Praat [35] obtaining good correlations for $G$ and $B$ traits. On [21] Mel-Frequency Spectrum Coefficients (MFCCs) were utilized obtaining 68\% and $63 \%$ of efficiency for Grade and Roughness $(R)$ traits, respectively, using Learning Vector Quantization (LVQ) methods for the pattern recognition stage but without any type of cross-validation techniques. The review of the state of the art reports that only [36] has used the same database and perceptual assessment used in the present study. The mentioned work proposed a set of complexity measurements and GMM to emulate a perceptual evaluation of all GRBAS traits, but its performance does not surpass $56 \%$ for $G$ or $R$.
In general, results seldom exceed $75 \%$ of efficiency; hence, there is still room for enhancement in the field of voice quality automatic evaluation. Thus, new parameterization approaches are needed and the use of Modulation spectrum (MS) emerges as a promising technique. MS provides a visual representation of sound energy spread in acoustic and modulation axes [37, 38] supplying information about perturbations related to amplitude and frequency modulation of the voice signal. Numerous acoustic applications use these spectra to extract features from audio signals from which some examples can be found in [39-42]. Although there are few publications centered in the characterization of dysphonic voices using this technique [11, 12, 23, 43], it can be stated that MS has not been studied deeply in the field of the detection of voice disorders and specially as a source of information to determine patient's degree of pathology. Some of the referred works have used MS to simulate an automatic perceptual analysis but, to the best of our knowledge, none of them offer well-defined parameters with a clear physical interpretation but transformations of MS which are not easily interpretable, limiting their application in the clinical practice.

The purpose of this work is to provide new parameters obtained from MS in a more reasoned manner, making them more comprehensible. The use of this spectrum and associated parameters as support indices is expected to be useful in medical applications since they provide easy-tounderstand information compared to others such as MFCC or complexity parameters, for instance. The new parameterization proposed in this work has been used as the input to a classification system that emulates a perceptual assessment of voice following the GRBAS scale in $G$ and $R$ traits. These two traits have been selected over the other three (Aesthenia $(A)$, Breathiness, and Strain $(S)$ ) since its assessment seems to be more reliable. De Bodt et al. [5] point that $G$ is the less unambiguously interpreted and $R$ has an intermediate reliability on its interpretation. These conclusions are coherent with those exposed in $[44,45]$. Similar findings are revealed in [6] which considers $R$ as one of the most reliable traits when using sustained vowel /ah : / as source of evaluation. It is convenient to specify that each feature of the GRBAS scale ranges from 0 to 3 , where 0 indicates no affection, 1 slightly affected, 2 moderately affected, and 3 severely affected voice regarding the corresponding trait. Thus evaluating according to this perceptual scale means developing different 4-class classifiers, one for each trait.

In this work, the results obtained with the proposed MSbased parameters are compared with a classic parameterization used to characterize voice in a wide range of applications: Mel-Frequency Cepstral Coefficients [46]. MFCCs have been traditionally used for speech and speaker recognition purposes since the last two decades and many works use these coefficients to detect voice pathologies with a good outcome.

The paper is organized as follows: Section 2 develops the theoretical background of modulation spectra features. Section 3 introduces the experimental setup and describes the database used in this study. Section 4 presents the obtained results. Lastly, Section 5 presents the discussions, conclusions, and future work. 


\section{Theoretical Background}

2.1. Modulation Spectra. This study proposes a new set of parameters based on MS to characterize the voice signal. MS provides information about the energy at modulation frequencies that can be found in the carriers of a signal. It is a three-dimensional representation where abscissa usually represents modulation frequency, ordinate axis depicts acoustic frequency, and applicate, acoustic energy. This kind of representation allows observing different voice features simultaneously such as the harmonic nature of the signal and the modulations present at fundamental frequency and its harmonics. For instance, the presence of tremor, understood as low frequency perturbations of the fundamental frequency, can be easily noticeable since it implies a modulation of pitch as an usual effect of laryngeal muscles improper activity. Other modulations associated with fundamental or harmonic frequencies could indicate the presence of a dysfunction of the phonatory system. Some examples can be found in [11].

To obtain MS, the signal is filtered using a short-time Fourier transform (sTFT) filter bank whose output is used to detect amplitude and envelope. This outcome is finally analyzed using FFT [47] producing a matrix $E$ where MS values at any point can be represented as $E\left(f_{a}, f_{m}\right)$. The columns at $E$ (fixed $f_{m}$ ) are modulation frequency bands, and rows (fixed $f_{a}$ ) are acoustic frequency bands. Therefore, $a$ can be interpreted as the index of acoustic bands and $m$, the index of modulation bands while $f_{a}$ and $f_{m}$ are the central frequencies of the respective bands. Due to the fact that values $E\left(f_{a}, f_{m}\right)$ have real and imaginary parts, the original matrix can be represented using the modulus $|E|$ and the phase $\arg (E)$ of the spectrum. Throughout this work, the MS has been calculated using the Modulation Toolbox library version 2.1 [48]. Some different configurations can be used to obtain $E$, where the most significant degrees of freedom are the use of coherent or noncoherent (Hilbert envelope) [49] modulation, the number of acoustic bands, and acoustic and modulation frequency ranges. The three-dimensional phase unwrapping techniques detailed in [50] are used to solve the phase ambiguity problems which appear when calculating $\arg \left(E\left(f_{a}, f_{m}\right)\right)$.

Figure 1 shows an example of MS extracted from two different voices on which the voice of a patient with gastric reflux, edema of larynx, and hyperfunction exhibits a more spread modulation energy in comparison to a normal voice.

However, one of the principal drawbacks of MS is that it provides a large amount of information that can not be easily processed automatically due to limitations of the existing pattern recognition techniques and voice disorders databases available. In this sense, MS matrices have to be processed to obtain a more compact but precise enough representation of the represented speech segments. Thus, after obtaining the MS, some representative parameters are extracted to feed a further classification stage. With this in mind, a new group of Morphological Parameters based on MS is proposed in this work: centroids [51] (MSC), dynamic range per band (DRB), Low Modulation Ratio (LMR), Dispersion Parameters (CIL, PALA, and RALA), Contrast (MSW), and Homogeneity
(MSH). All these parameters use the MS modulus as input source, except the last two which also use the phase.

2.1.1. Centroids (MSC) and Dynamic Range per Band (DRB). Centroids provide cues about the acoustic frequency that represents the central energy or the energy center at each modulation band. To obtain MSC, MS modulus is reduced to an absolute number of modulation bands usually ranging from 4 to 26, each containing information about the modulation energy in that band along the acoustic frequency axis. Once the reduced MS is computed, centroids are calculated following the expression

$$
\operatorname{MSC}\left(f_{m}\right)=\frac{\sum_{a} f_{a} \cdot\left|E\left(f_{a}, f_{m}\right)\right|}{f_{\text {pitch }} \cdot \sum_{a}\left|E\left(f_{a}, f_{m}\right)\right|},
$$

where $f_{a}$ and $f_{m}$ represent the central frequency of the acoustic and modulation bands, respectively, and $f_{\text {pitch }}$ is the pitch frequency.

As a matter of example, Figure 2 depicts a representation of MSC extracted from a MS.

Once MS is reduced to a small number of modulation bands, the dynamic range is calculated for every band (DRB) as the difference between the highest and the lowest levels in the band. These parameters provide information about the flatness of the MS depending on the modulation frequency.

2.1.2. Low Modulation Ratio (LMR). LMR, expressed in $\mathrm{dB}$, is the ratio between energy in the first modulation band $\varepsilon\left(f_{a\left(f_{\text {pitch }}\right)}, f_{1}\right)$ at acoustic frequency $f_{\text {pitch }}$ and the global energy in all modulation bands covering at least from 0 to $25 \mathrm{~Hz}$ at acoustic frequency $f_{\text {pitch }}, \varepsilon\left(f_{a\left(f_{\text {pitch }}\right)}, f_{m(25 \mathrm{~Hz})}\right)$. Its calculation is carried out according to the following expressions. These bands are represented in Figure 3:

$$
\operatorname{LMR}=10 \cdot \log \left(\frac{\varepsilon\left(f_{a\left(f_{\text {pitch }}\right)}, f_{1}\right)}{\varepsilon\left(f_{a\left(f_{\text {pitch }}\right)}, f_{m(25 \mathrm{~Hz})}\right)}\right)
$$

being

$$
\varepsilon\left(f_{a}, f_{k}\right)=\sum_{m=1}^{k}\left|E\left(f_{a}, f_{m}\right)\right|^{2},
$$

where $a\left(f_{\text {pitch }}\right)$ is the index of the acoustic band including pitch frequency and $m(25 \mathrm{~Hz})$, the index of the modulation band including $25 \mathrm{~Hz}$.

The $0-25 \mathrm{~Hz}$ band has been selected to represent all possible cases of tremor and low frequency modulations around pitch frequency $[52,53]$.

2.1.3. Contrast and Homogeneity. Representing MS (modulus or phase) as two-dimensional images let observe that pathological voices usually have more complex distributions. Images related to normal voices are frequently more homogeneous and present less contrast, as can be seen in Figure 1. Accordingly, Homogeneity and Contrast are used as two MS features since they provide information about the existence of voice perturbations. 


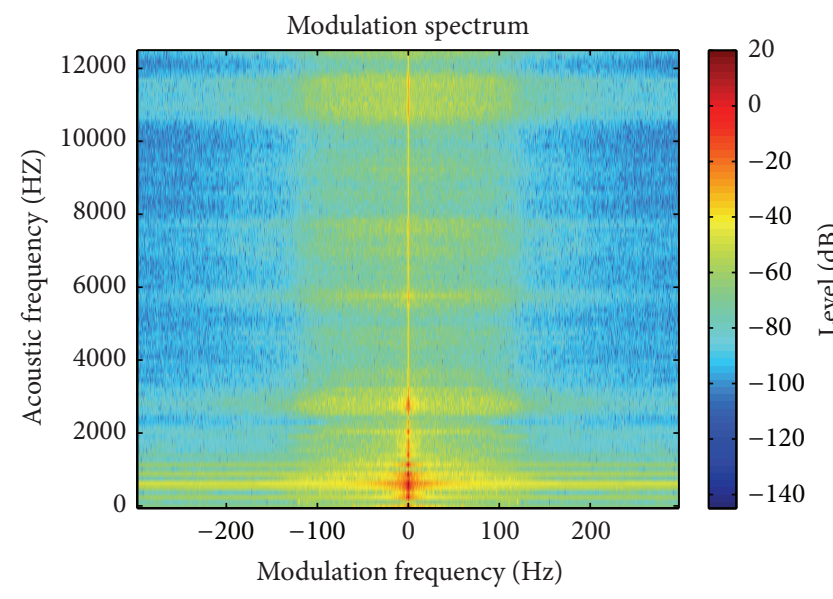

(a)

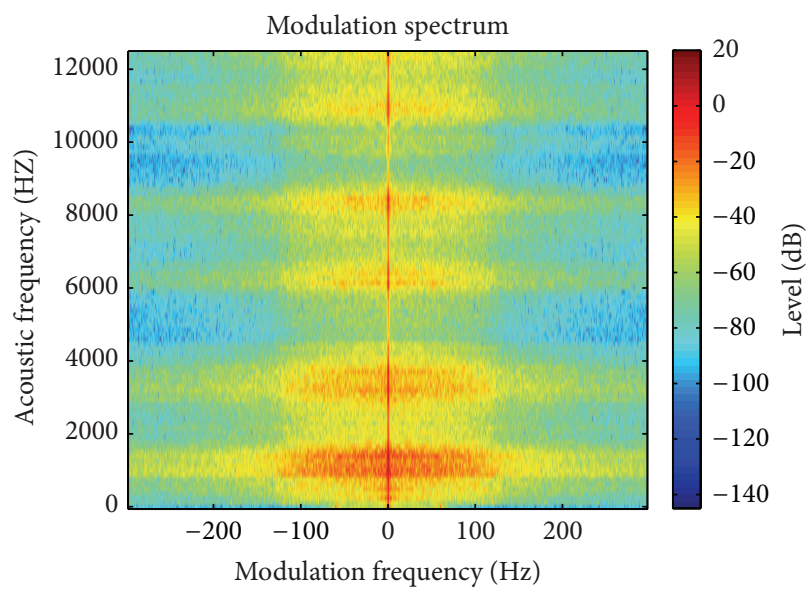

(b)

FIGURE 1: MS modulus of a normal voice (a) and pathological voice of a patient with gastric reflux, edema of larynx, and hyperfunction (b).

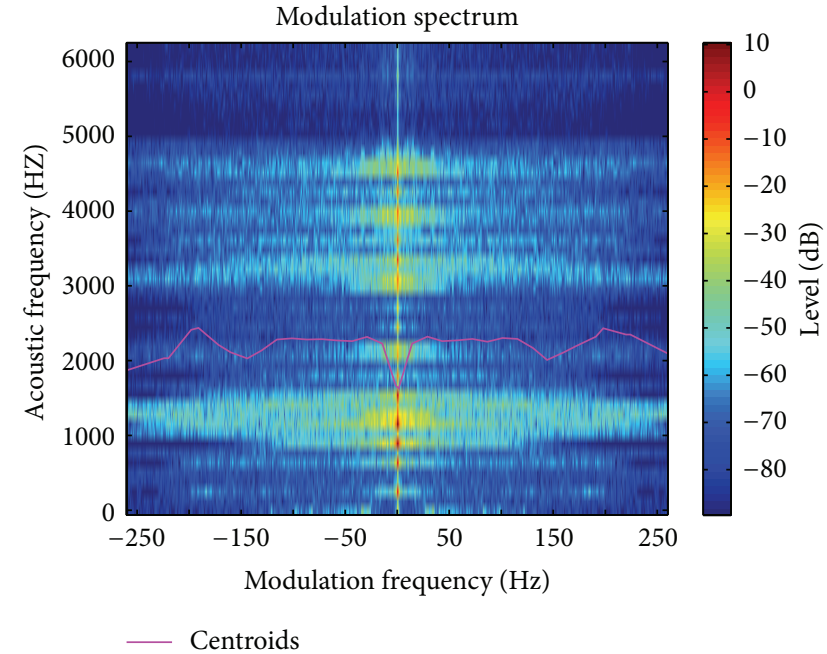

(a)

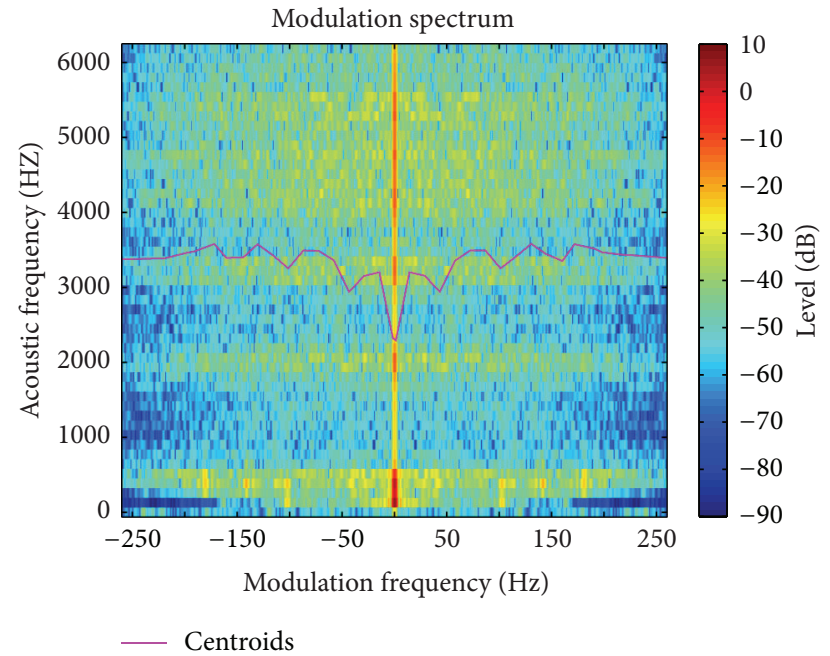

(b)

FIGURE 2: MS centroids of a normal voice (a) and a pathological voice (b) of a patient with gastric reflux, keratosis, and laryngocele.

Homogeneity is computed using the Bhanu method described by the following expression, as stated in [54]:

$$
\mathrm{MSH}=\sum_{a} \sum_{m}\left[E\left(f_{a}, f_{m}\right)-{\overline{E\left(f_{a}, f_{m}\right)}}_{3 \times 3}\right]^{2},
$$

with MSH being the MS Homogeneity value; $E\left(f_{a}, f_{m}\right)$ the modulation spectra computation (modulus or phase) at point $\left(f_{a}, f_{m}\right)$; and $\overline{E\left(f_{a}, f_{m}\right)_{3 \times 3}}$ the average value in a $3 \times 3$ window centered at the same point.

Contrast is computed using a variation of the WeberFechner contrast relationship method described by the following expression as stated in [54]:

$$
\operatorname{MSW}\left(f_{a}, f_{m}\right)=\sum_{a^{\prime}} \sum_{m^{\prime}} C_{f_{a}, f_{m}},
$$

where

$$
C_{f_{a}, f_{m}}=\frac{\left|E\left(f_{a}, f_{m}\right)-E\left(f_{a^{\prime}}, f_{m^{\prime}}\right)\right|}{\left|E\left(f_{a}, f_{m}\right)+E\left(f_{a^{\prime}}, f_{m^{\prime}}\right)\right|}
$$

representing $\left(f_{a^{\prime}}, f_{m^{\prime}}\right)$ the vertical and horizontal adjacent points to $\left(f_{a}, f_{m}\right)$. The global MSW is considered the sum of all points in $\operatorname{MSW}\left(f_{m}, f_{a}\right)$ divided by the total number of points to normalize.

The MS used to calculate MSH and MSW at each point of the matrix is represented in Figure 3.

2.1.4. Dispersion Parameters. As MS differs from normal to pathological voices, changes in the histograms of MS modulus reflect the effects of a dysfunction in a patient's voice. A short view to the MS permits to observe that voices with high $G$ and $R$ traits usually have a larger number of points with levels above the average value of $\left|E\left(f_{m}, f_{a}\right)\right|$. The level of 


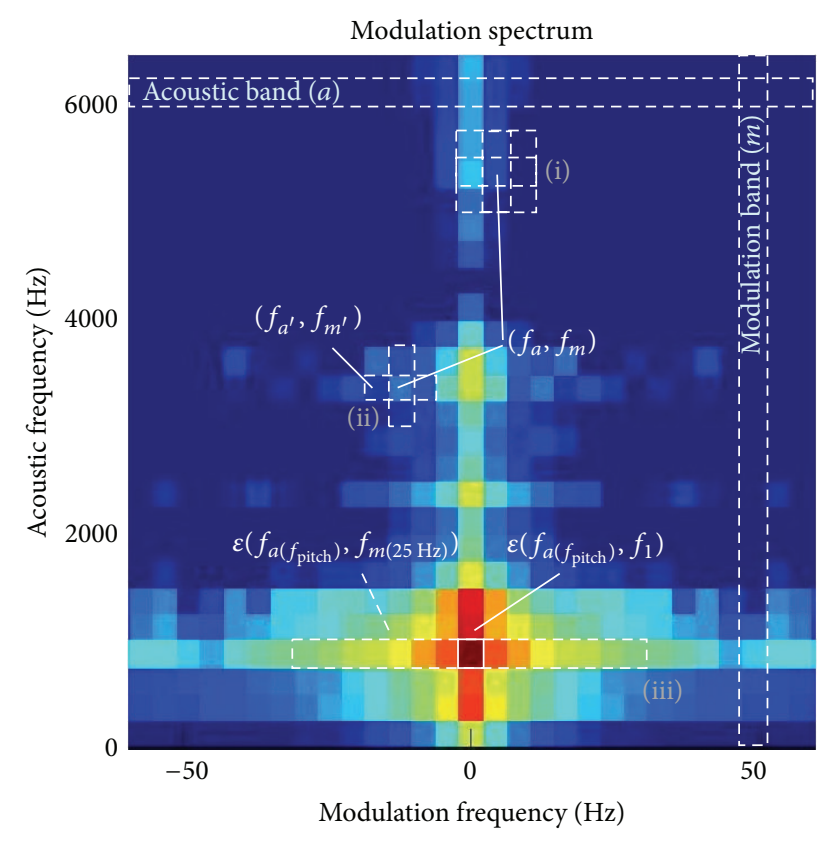

Figure 3: Points of the MS matrix used to obtain MSH (i), MSW (ii), and LMR (iii).

these points can be interpreted as the dispersion of the energy present in the central modulation band $(0 \mathrm{~Hz})$ towards side bands respecting the case of a normal voice.

With this in mind, three Morphological Parameters are proposed to measure such dispersion effect: Cumulative Intersection Level (CIL), Normalized Number of Points above Linear Average (PALA), and Ratio of Points above Linear Average (RALA). CIL is the intersection between the histogram increasing and decreasing cumulative curves. Histogram is processed from MS modulus in logarithmic units (dB). As shown in Figure 4, CIL tends to be higher in pathological than in healthy voices. In that case, the difference is $19 \mathrm{~dB}$. On the other hand, PALA is the number of points in MS modulus which are above average (linear units) divided by the total number of points of MS. RALA is quite similar to PALA but in this case it represents the ratio of points in MS modulus which are over the average and the number of points which are above this average instead of the total number of points in $E\left(f_{a}, f_{m}\right)$. Calculation of PALA and RALA is detailed in the following expressions:

$$
\begin{aligned}
\text { PALA } & =\frac{\mathrm{NA}}{\mathrm{NT}}, \\
\text { RALA } & =\frac{\mathrm{NA}}{\mathrm{NB}}
\end{aligned}
$$

being

$$
\begin{aligned}
& \mathrm{NA}=\sum_{f_{a}} \sum_{f_{m}} \gamma\left(f_{a}, f_{m}\right), \\
& \mathrm{NB}=\sum_{f_{a}} \sum_{f_{m}} 1-\gamma\left(f_{a}, f_{m}\right),
\end{aligned}
$$

$$
\gamma\left(f_{a}, f_{m}\right)= \begin{cases}1 & \left|E\left(f_{a}, f_{m}\right)\right| \geq \overline{|E|} \\ 0 & \left|E\left(f_{a}, f_{m}\right)\right|<\overline{|E|},\end{cases}
$$

where $\overline{|E|}$ is the MS modulus average, NA the number of points above $\overline{|E|}$, NB the number of points below $\overline{|E|}$, and NT the total number of points in $E\left(f_{a}, f_{m}\right)$.

In the cases in which the number of points above linear average increases, the difference between PALA and RALA increases too as the denominator in PALA stays constant and the denominator in RALA decreases. Figure 5 represents these points in a healthy and a pathological voice. It is noticeable that, as expected, the MS of dysphonic voices presents more points above the modulus average.

\section{Experimental Setup}

3.1. Database. The Kay Elemetrics Voice Disorders Database recorded by the Massachusetts Eye and Ear Infirmary Voice Laboratory (MEEI) was used for this study [55] due to its commercial availability. The database contains recordings of the phonation of the sustained vowel / $a h$ : / (53 normal, 657 pathological) and utterances corresponding to continuous speech during the reading of the "Rainbow passage" (53 normal, 661 pathological). The sample frequency of the recordings is $25 \mathrm{kHz}$ with a bit depth of 16 bits. From the original amount of speakers recorded in the database, a first corpus of 224 speakers was selected according to the criteria found in [56] being named henceforward as the original subset. The utterances corresponding to the sustained vowel and the continuous speech recordings were used to rate $G$ and $R$ for each patient according to the GRBAS scale. The degree of these traits has been estimated three times by two speech therapists. One of them evaluated the whole database once, and the second one performed the assessment twice in two different sessions. Regarding this study, only the sustained vowels are considered. With the aim of obtaining more consistent labels, two reduced subsets of 87 and 85 audio files for $G$ and $R$, respectively, were considered. Those files are chosen from the initial corpus of 224 recordings on the basis of selecting only those whose labeling was in a total agreement for the three assessments making up the $G$ and $R$ agreement subsets. This reduction was performed to avoid modeling inter/intraraters variability inherent to the process of assigning perceptual labels to each speaker. In any case, all tests were performed for the three subsets to provide evidences about such reduction. Some statistics of database are shown in Table 1.

With the aim of sharing relevant information and to promote a more reliable comparison of techniques and results, the names of the recordings extracted from MEEI corpus that were used for this study along with their respective $G$ and $R$ levels are included in Appendix, Table 6.

3.2. Methodology. One of the purposes of this work is to test a new source of parameters to characterize voice perturbations by replicating clinician's $G$ and $R$ perceptual evaluations. 
TABLE 1: Subsets statistics.

\begin{tabular}{|c|c|c|c|c|c|c|}
\hline \multirow{2}{*}{ Subset name } & \multicolumn{2}{|c|}{ Number of subjects } & \multicolumn{2}{|c|}{ Age range } & \multicolumn{2}{|c|}{ Average age } \\
\hline & Female & Male & Female & Male & Female & Male \\
\hline Original (226 files) & 90 & 134 & $21-52$ & $26-59$ & $35.8 \pm 8.2$ & $39.9 \pm 9.1$ \\
\hline Agreement- $G$ (87 files) & 52 & 35 & $24-52$ & $26-58$ & $36.6 \pm 7.6$ & $39.5 \pm 9.7$ \\
\hline Agreement- $R$ (85 Files) & 51 & 34 & $22-52$ & $26-58$ & $35.4 \pm 7.6$ & $37.9 \pm 9.2$ \\
\hline
\end{tabular}

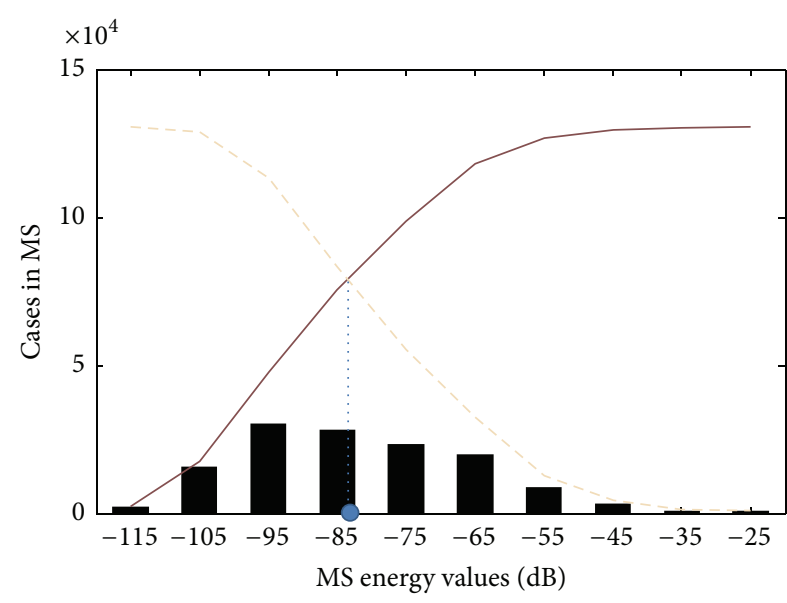

MS histogram
Inc. cumulative - CIL

(a)

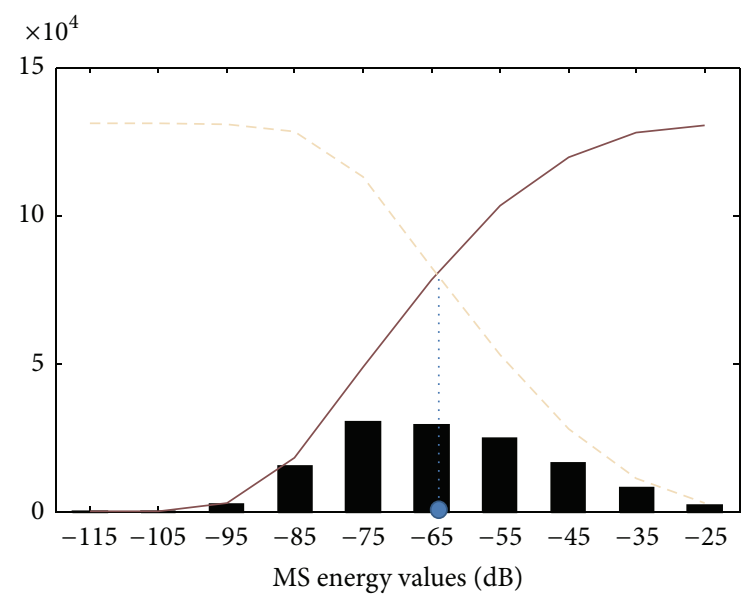

$\begin{array}{lll}\text { MS histogram } & & \text { Dec. cumulative } \\ \text { Inc. cumulative } & \mathrm{CIL}\end{array}$

(b)

FIGURE 4: CIL calculation in a normal voice (top) and a pathological voice (bottom) diagnosed of bilateral laryngeal tuberculosis.

So as to quantify the contribution of this new approach, a baseline parameterization has been established to compare with the novel one. Consequently, all tests are performed using the parameters of the baseline system (MFCCs) and the MS Morphological Parameters. A large number of tests were accomplished to find the best setting, modifying the number of centroids or the frame duration among other degrees of freedom.

The methodology employed in this paper is shown in Figure 6, while each one of its stages is explained next. Basically, it is the classical supervised learning arrangement, which can be addressed using either classification or regression techniques. For the sake of simplicity and to concentrate on the novel parameterization approach, a simple Gaussian Mixture Model (GMM) classification back-end was employed to recognize the presence of the perturbations in the voice signal which presumably would produce high levels of $G$ and $R$ during perceptual analysis.

3.2.1. Characterization. Two parameterization approaches are considered in this study: MFCCs and MS Morphological Parameters. The MFCCs are the ground of the baseline system and were used for comparison due to their wide use in speech technology applications.

The MFCCs are calculated following a method based on the human auditory perception system. The mapping between the real frequency scale $(\mathrm{Hz})$ and the perceived frequency scale (mels) is approximately linear below $1 \mathrm{kHz}$ and logarithmic for higher frequencies. Such mapping converts real into perceived frequency. In this work MFCCs are estimated using a nonparametric FFT-based approach. Coefficients are obtained by calculating the Discrete Cosine Transform (DCT) over the logarithm of the energy in several frequency bands. The bandwidth of the critical band varies according to the perceived frequency. Each band in the frequency domain is bandwidth dependant of the filter central frequency. The higher the frequency is, the wider the bandwidth is. To obtain these parameters, a typical setup of 30 triangular filters and cepstral mean subtraction was used. Their computation is carried out over speech segments framed and windowed using Hamming windows overlapped $50 \%$. Duration of frames oscillates from 20 to $100 \mathrm{~ms}$ in $20 \mathrm{~ms}$ steps. For the sake of comparison the number of MFCCs ranges from 10 to 22 coefficients. 0'th order cepstral coefficient is removed.

Regarding the MS Morphological Parameters, each signal is also framed and windowed using Hamming windows overlapped $50 \%$. The window lengths are varied in the range of 20-200 ms in $20 \mathrm{~ms}$ steps. The feature vector extracted from MS is composed of the following: MSC, DRB, LMR, MSW, MSH, CIL, PALA, and RALA. The number of bands to obtain centroids and dynamic range features is varied in the range of $[6,22]$ with a step size of 2 . Considering that MSW and MSH provide two features each (one for modulus and other for phase), the feature vector corresponding to each frame ranges from 20 to 44 values before using data reduction 


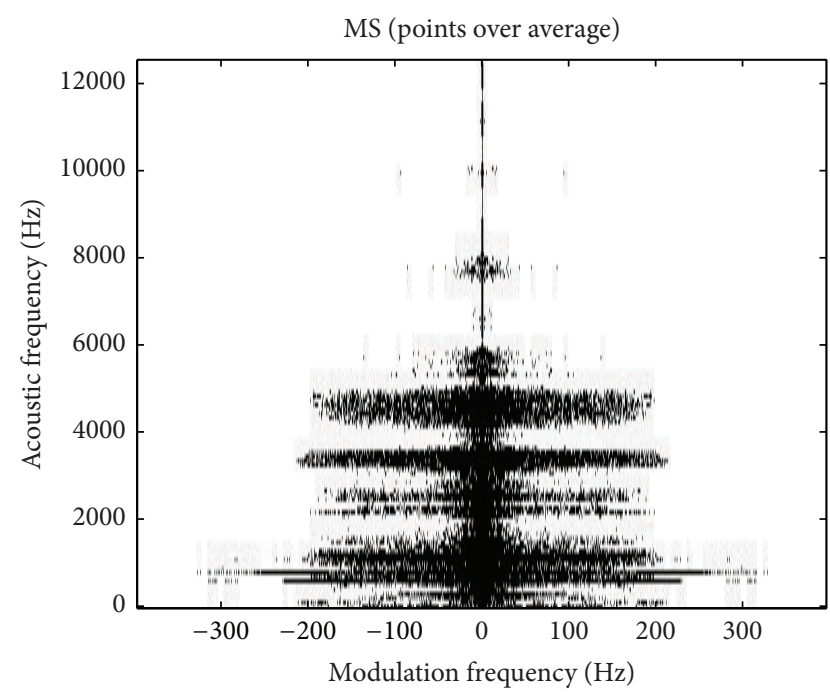

(a)

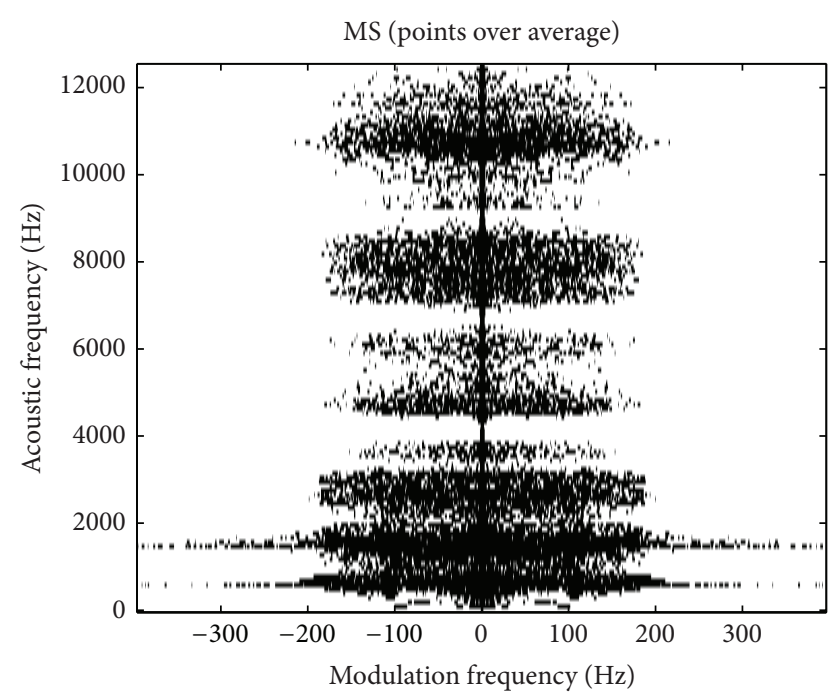

(b)

FIGURE 5: Points above (black) and below (white) modulus average in MS for a normal voice (a) PALA = 0.11, RALA =0.12, and a pathological voice due to bilateral laryngeal tuberculosis $(b)$ PALA $=0.21$, RALA $=0.27$.

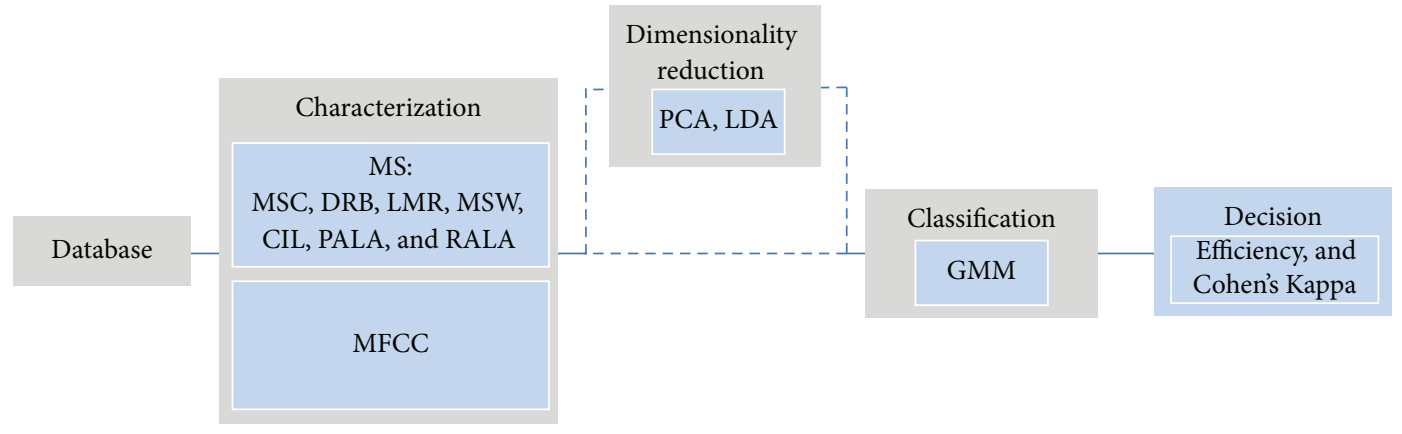

FIgURE 6: Outline of the automatic detector presented in the paper.

techniques. Both, coherent and noncoherent modulation (Hilbert envelope) were used for testing separately. Acoustic frequency $\operatorname{span}[0-12.5 \mathrm{kHz}]$ is divided into 128 bands and maximum modulation frequency varied from 70 to $500 \mathrm{~Hz}$ to allow different configurations during tests.

In addition, first derivative $(\Delta)$ and second derivative $(\Delta \Delta)$, representing the speed and acceleration in the changes of every characteristic, are added to the features in order to include interframe attributes [46]. The calculation of $\Delta$ and $\Delta \Delta$ was carried out employing finite impulse response filters using a length of 9 samples to calculate $\Delta$ and 3 in the case of $\Delta \Delta$.

All these features are used to feed a subsequent classification phase in two different ways depending on the test: some experiments are accomplished using features as they are obtained, and others use a reduced version to relieve the curse of dimensionality effect.

In the dimensionality reduction stage, PCA [57] and LDA [58] techniques are used varying the dimension of the feature vectors used for classification. In the case of LDA, all feature vectors are reduced to a 3 -dimensional space. Concerning
PCA, reduction ranges from 80 to $95 \%$. With respect to these techniques, only the training data set is used to obtain the models which are employed to reshape all the data: training and test data sets. This process is repeated for every iteration of the GMM training-test process carried out for validation. The dimensionality reduction is applied for both MS Morphological Parameters and MFCCs features with and without derivatives separately.

3.2.2. Validation. Following the characterization, a LeaveOne-Out (LOO) cross-validation scheme [33] was used for evaluating the results. On this scheme one file is considered for testing and the remaining files of the database are used as training data, generating what is called a fold. As a result, there are as many folds as number of files, and each of them will provide a classification accuracy. The global result for a certain parameterization experiment is the average of the results in all folds. In spite of having a higher computational cost, this cross-validation technique has been selected instead of other less computationally costly such as $k$-folds [59] due 
TABLE 2: Results expressed as efficiency \pm confidence interval and Cohen’s Kappa Index using MFCCs and MS features.

\begin{tabular}{|c|c|c|c|c|c|c|c|c|}
\hline \multirow{3}{*}{ Features } & \multicolumn{4}{|c|}{ Original subset } & \multicolumn{4}{|c|}{ Agreement subset } \\
\hline & \multicolumn{2}{|c|}{$G$} & \multicolumn{2}{|c|}{$R$} & \multicolumn{2}{|c|}{$G$} & \multicolumn{2}{|c|}{$R$} \\
\hline & Efficiency (\%) & $\kappa$ & Efficiency (\%) & $\kappa$ & Efficiency (\%) & $\kappa$ & Efficiency (\%) & $\kappa$ \\
\hline MFCC & $54.5 \pm 6.5$ & 0.37 & $53.1 \pm 6.5$ & 0.51 & $75.9 \pm 9.0$ & 0.64 & $76.5 \pm 9.0$ & 0.64 \\
\hline $\mathrm{MFCC}+\mathrm{PCA}$ & $56.3 \pm 6.5$ & 0.39 & $52.2 \pm 6.6$ & 0.31 & $78.2 \pm 8.7$ & 0.67 & $74.1 \pm 9.3$ & 0.60 \\
\hline MFCC + LDA & $45.5 \pm 6.5$ & 0.27 & $48.2 \pm 6.6$ & 0.29 & $65.5 \pm 10.0$ & 0.48 & $68.3 \pm 9.9$ & 0.50 \\
\hline MS & $60.3 \pm 6.4$ & 0.45 & $54.9 \pm 6.5$ & 0.36 & $81.6 \pm 8.1$ & 0.72 & $76.5 \pm 9.0$ & 0.63 \\
\hline $\mathrm{MS}+\mathrm{PCA}$ & $58.5 \pm 6.5$ & 0.43 & $58.0 \pm 6.5$ & 0.41 & $79.3 \pm 8.5$ & 0.69 & $78.8 \pm 8.7$ & 0.68 \\
\hline $\mathrm{MS}+\mathrm{LDA}$ & $58.9 \pm 6.4$ & 0.44 & $59.8 \pm 6.4$ & 0.43 & $81.6 \pm 8.1$ & 0.72 & $83.5 \pm 7.9$ & 0.74 \\
\hline
\end{tabular}

TABLE 3: Results expressed as efficiency \pm confidence interval and Cohen's Kappa Index for MFCCs and MS features including $\Delta$ and $\Delta \Delta$.

\begin{tabular}{|c|c|c|c|c|c|}
\hline \multirow{3}{*}{ Features } & \multirow{3}{*}{ Dimensionality reduction } & \multicolumn{4}{|c|}{ Agreement subset } \\
\hline & & \multicolumn{2}{|c|}{$G$} & \multicolumn{2}{|c|}{$R$} \\
\hline & & Efficiency (\%) & $\kappa$ & Efficiency (\%) & $\kappa$ \\
\hline \multirow{2}{*}{$\mathrm{MFCC}+\Delta$} & PCA & $78.2 \pm 8.7$ & 0.67 & $74.1 \pm 9.3$ & 0.60 \\
\hline & LDA & $72.4 \pm 9.4$ & 0.58 & $58.8 \pm 10.5$ & 0.35 \\
\hline \multirow{2}{*}{$\mathrm{MFCC}+\Delta+\Delta \Delta$} & PCA & $80.5 \pm 8.3$ & 0.71 & $77.7 \pm 8.8$ & 0.66 \\
\hline & LDA & $72.4 \pm 9.4$ & 0.58 & $62.4 \pm 10.3$ & 0.41 \\
\hline \multirow{2}{*}{$\mathrm{MS}+\Delta$} & PCA & $81.6 \pm 8.1$ & 0.73 & $80.0 \pm 8.5$ & 0.69 \\
\hline & LDA & $80.5 \pm 8.3$ & 0.72 & $81.2 \pm 8.3$ & 0.71 \\
\hline \multirow{2}{*}{$\mathrm{MS}+\Delta+\Delta \Delta$} & PCA & $79.3 \pm 8.5$ & 0.63 & $80.0 \pm 8.5$ & 0.70 \\
\hline & LDA & $80.5 \pm 8.3$ & 0.71 & $84.7 \pm 7.7$ & 0.76 \\
\hline
\end{tabular}

to its suitability in view of the reduced number of recordings contained in the agreement subsets.

3.2.3. Classification. The features extracted during the parameterization stage are used to feed the classifier, which is based on the Gaussian Mixture Model (GMM) paradigm. Having a data vector $\mathbf{x}$ of dimension $d$ resulting from the parameterization stage, a GMM is a model of the probability density function defined as a finite mixture of $g$ multivariate Gaussian components of the form:

$$
p\left(\mathbf{x} \mid \Theta_{i}\right)=\sum_{r=1}^{g} \lambda_{r} \mathcal{N}\left(\mathbf{x} ; \boldsymbol{\mu}_{r}, \boldsymbol{\Sigma}_{r}\right),
$$

where $\lambda_{r}$ are scalar mixture weights, $\mathcal{N}(\cdot)$ are Gaussian density functions with mean $\boldsymbol{\mu}_{r}$ of dimension $d$ and covariances $\boldsymbol{\Sigma}_{r}$ of dimension $d \times d$, and $\Theta_{i}=\left.\left\{\boldsymbol{\lambda}_{r}, \boldsymbol{\mu}_{r}, \boldsymbol{\Sigma}_{r}\right\}\right|_{r=1} ^{g}$ comprises the abovementioned set of parameters that defines the class to be modeled. Thus, for each class $\Theta_{i}$ to be modeled (i.e., values of the $G$ and $R$ perceptual levels: $0,1,2$, or 3), a GMM is trained. $\Theta_{i}$ is estimated using the expectation-maximization algorithm (EM) [60]. The final decision about the class that a vector belongs to is taken establishing for each pair of classes $i, j$ a threshold $\Gamma$ over the likelihood ratio (LR), that in the logarithmic domain is given by

$$
\operatorname{LR}=\log \left(p\left(\mathbf{x} \mid \Theta_{i}\right)\right)-\log \left(p\left(\mathbf{x} \mid \Theta_{j}\right)\right)
$$

The threshold $\Gamma$ is fixed at the Equal Error Rate (ERR) point.
In this stage, the number of Gaussian components of the GMM was varied from 4 to 48 . The assessment of the classifier was performed by means of efficiency and Cohen's Kappa Index $(\kappa)$ [61]. This last indicator provides information about the agreement between the results of the classifier and the clinician's perceptual labeling.

\section{Results}

The best results obtained for each type of test can be observed in Table 2, which disposes the outcomes taking into account the type of characterization, dimensionality reduction, and database subset used. All tests were performed using the aforementioned sets of the database with and without PCA and LDA techniques. Table 3 shows the outcomes adding first and second derivative to the original parameterizations before dimensionality reduction. All results are expressed in terms of efficiency and Cohen's Kappa Index. For the sake of simplicity, only results obtained with the third labeling of the original subset are shown, corresponding to columns G3 and $R 3$ in Appendix, Table 6.

Concerning $G$ trait, absolute best results (81.6\%) are obtained in the agreement database, using MS $+\Delta$ in $140 \mathrm{~ms}$ frames, 22 centroids, Hilbert envelope, $240 \mathrm{~Hz}$ as max. modulation frequency, dimensionality reduction through PCA (93\% reduction), and 4 GMM. Respecting MFCC, best results are obtained using MFCCs $+\Delta+\Delta \Delta, 22$ coefficients, PCA, $20 \mathrm{~ms}$ frames, and 8 GMM.

Relating to $R$, as expected, absolute best results $(84.7 \%)$ are also obtained in the agreement database using MS + 
TABLE 4: Confusion matrices related to absolute best results in MS parameters and MFCCs. $G_{T}$ and $R_{T}$ are target labels while $G_{P}$ and $R_{P}$ are predicted labels.

\begin{tabular}{cccccccccc}
\hline \multicolumn{1}{c}{ Grade } \\
& $G_{P} 0$ & $G_{P} 1$ & $G_{P} 2$ & $G_{P} 3$ & $R_{P} 0$ & $R_{P} 1$ & $R_{P} 2$ & $R_{P} 3$ \\
\hline \multicolumn{1}{c}{ MS Morphological Parameters } \\
$G_{T} 0$ & $\mathbf{2 8}$ & 1 & 0 & 1 & $R_{T} 0$ & $\mathbf{3 8}$ & 1 & 0 & 0 \\
$G_{T} 1$ & 3 & $\mathbf{3}$ & 1 & 0 & $R_{T} 1$ & 3 & $\mathbf{1}$ & 2 & 0 \\
$G_{T} 2$ & 1 & 1 & $\mathbf{1 1}$ & 1 & $R_{T} 2$ & 1 & 0 & $\mathbf{1 3}$ & 1 \\
$G_{T} 3$ & 3 & 0 & 4 & $\mathbf{2 9}$ & $R_{T} 3$ & 3 & 1 & 1 & $\mathbf{2 0}$ \\
\hline \multicolumn{10}{c}{ MFCCs } \\
$G_{T} 0$ & $\mathbf{2 7}$ & 0 & 2 & 1 & $R_{T} 0$ & $\mathbf{3 5}$ & 0 & 1 & 3 \\
$G_{T} 1$ & 2 & $\mathbf{0}$ & 5 & 0 & $R_{T} 1$ & 3 & $\mathbf{0}$ & 3 & 0 \\
$G_{T} 2$ & 0 & 0 & $\mathbf{1 3}$ & 1 & $R_{T} 2$ & 1 & 0 & $\mathbf{9}$ & 5 \\
$G_{T} 3$ & 0 & 0 & 6 & $\mathbf{3 0}$ & $R_{T} 3$ & 1 & 0 & 2 & $\mathbf{2 2}$ \\
\hline
\end{tabular}

TABLE 5: Altman interpretation of Cohen's index.

\begin{tabular}{lc}
\hline$\kappa$ & Agreement \\
\hline$\leq 0.20$ & Poor \\
$0.21-0.40$ & Fair \\
$0.41-0.60$ & Medium \\
$0.61-0.80$ & Good \\
$0.81-1.00$ & Excellent \\
\hline
\end{tabular}

$\Delta+\Delta \Delta$ calculated in $100 \mathrm{~ms}$ frames, 14 centroids, Hilbert envelope, $240 \mathrm{~Hz}$ as max. modulation frequency, dimensionality reduction through LDA, and 16 GMM. Respecting MFCC, best results are obtained using MFCCs $+\Delta+\Delta \Delta, 22$ coefficients, PCA, $20 \mathrm{~ms}$ frames, and $48 \mathrm{GMM}$.

Table 4 shows confusion matrices for MFCC and MS Morphological Parameters as the sum of the confusion matrices obtained at each of the test folds. They are calculated using the mentioned configurations that leaded to the best results.

\section{Conclusion and Discussions}

This study presents a new set of parameters based on MS being developed to characterize perturbations of the human voice. The performance of these parameters has been tested with an automatic system that emulates a perceptual assessment according to the $G$ and $R$ features of the GRBAS scale. The proposed automatic system follows a classical supervised learning setup, based on GMM. The outcomes have been compared to those obtained with a baseline setup using the classic MFCCs as input features. Dimensionality reduction methods as LDA and PCA have been applied to mitigate the curse of dimensionality effects induced by the size of the corpus used to train and validate the system. Best results are obtained with the proposed MS parameters, providing $81.6 \%$ and $84.7 \%$ of efficiency and 0.73 and 0.76 Cohen's Kappa Index for $G$ and $R$, respectively, in the agreement subset. Having in mind Altman interpretation of Cohen's index [62], shown in Table 5, the agreement can be considered "good", almost "excellent." Likewise, most errors raised by the system correspond with adjacent classes, as it can be deduced from the confusion matrices represented in Table 4. It is noticeable that in many cases the second class (level 1 in traits $G$ and $R$ ) is not detected properly and the main reason may be the lack of subjects of class 2 (level 1 in $G$ and $R$ ) in the used corpus. The fact that GMM classifiers were trained with a poor quantity of class 2 frames with respect to the other classes explains the higher percentage of errors obtained for this class. In order to solve this problem in future works it might be necessary to use classification techniques for imbalanced data [63]. Another possible reason for the mismatching of intermediate classes ( $G$ and $R$ equal to 1 or 2 ) is that these are the less reliable levels in GRBAS perceptual assessment as it was described by de Bodt et al. [5].

In reference to the outcomes obtained with features without dimensionality reduction, results are better for the agreement subsets using MS Morphological Parameters. Moreover, when applying LDA to the MS feature space, an absolute improvement of a $9 \%$ is obtained for $R$ in comparison to MFCCs, leading to the best absolute outcome obtained and denoting that the MS Morphological Parameters are in some sense linearly separable. As a starting point, most of the agreement subset tests were performed with what we have called the original subset (224 files) using the three available label groups separately: one of them generated by one of the speech therapists and the other two created by the other specialist in two different sessions. In these cases, in spite of having a higher number of files and a more class-balanced database, results barely exceed $60 \%$ of efficiency. This demonstrates that the consistency of the database labeling (i.e., removing the noise introduced during the labeling process due to intraand interrater's variability) is crucial to obtain more accurate results. An interesting conclusion is that further studies should utilize only consistent labels obtained in agreement with several therapists and in different evaluation sessions.

In order to search for some evidences proving that the selected cross-validation technique is not influencing the results by producing corpus-adjusted trained models, most of the tests are launched again using a 6-fold crossvalidation technique as a prospecting experiment. Almost the same maximum efficiencies were obtained in all cases with a difference of around $\pm 1 \%$, suggesting that the selected cross-validation technique is not producing corpus-adjusted trained models.

Regarding the use of derivatives $\Delta$ and $\Delta \Delta$, they improve performance mainly when using MFCCs in $20 \mathrm{~ms}$ frames for $G$ trait. This suggests that derivatives provide relevant extra information related to the short term variations occurred in pathological voices [64]. In the rest of the cases the improvements are limited; therefore, the influence of derivatives in $G$ and $R$ detection systems should be studied in detail in the future work.

Comparing this work with other studies mentioned in Section 1, results with MFCCs are coherent with these obtained in [17, 21,36], although methodologies followed in them are different to the one proposed in this study. As it is stated in Section 1, previous studies seldom exceed 75\% efficiency. Taking into account $G$ and $R$ traits, only $[19,20]$ surpass that value achieving $80 \%$ for $G$ trait. 
TABLE 6: Subsets labeling.

\begin{tabular}{|c|c|c|c|c|c|c|c|c|c|c|c|c|c|c|c|c|c|c|c|c|c|c|c|c|c|c|c|}
\hline File & G1 & G2 & G3 & $R 1$ & $R 2$ & R3 & File & G1 & G2 & G3 & $R 1$ & $R 2$ & R3 & File & G1 & G2 & G3 & $R 1$ & $R 21$ & $R 3$ & File & G1 & G2 & G3 & $R 1$ & $R 2$ & R3 \\
\hline alb18an & 2 & 3 & 3 & 2 & 3 & 3 & pclnal & 0 & 0 & 0 & 0 & 0 & 0 & lbal5an & 2 & 0 & 0 & 1 & 0 & 0 & nc26an & 2 & 3 & 3 & 2 & 2 & 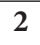 \\
\hline amcl4an & 1 & 3 & 3 & 1 & 3 & 3 & gsbllan & 3 & 3 & 3 & 3 & 3 & 3 & lba24an & 2 & 0 & 0 & 1 & 0 & 0 & md25an & 2 & 3 & 2 & 2 & 3 & 2 \\
\hline aos21an & 3 & 3 & 3 & 3 & 3 & 3 & gxl21an & 1 & 0 & 0 & 1 & 0 & 0 & dplnal & 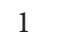 & 0 & 0 & 8 & 0 & 0 & mf03an & 2 & 1 & 1 & 2 & 1 & \\
\hline axd19an & 0 & $\mathbf{0}$ & $\mathbf{0}$ & 0 & $\mathbf{0}$ & $\mathbf{0}$ & gxt10an & 3 & 3 & 3 & 3 & 3 & 3 & les15an & 3 & 3 & 3 & 3 & 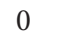 & 0 & rccllan & 2 & 3 & 3 & 2 & 2 & 2 \\
\hline axhlnal & 0 & $\mathbf{0}$ & $\mathbf{0}$ & 0 & $\mathbf{0}$ & $\mathbf{0}$ & gzzlnal & 1 & 0 & 0 & 1 & 0 & 0 & lgm01an & 1 & 0 & 0 & 1 & 0 & 0 & rhglnal & 0 & 1 & 0 & 0 & 1 & \\
\hline axt13an & 1 & 1 & 1 & 1 & 0 & 1 & hbllnal & 1 & 0 & 0 & 1 & 0 & 0 & ljh06an & 2 & 2 & 2 & 2 & 2 & 2 & rhmlnal & $\mathbf{0}$ & 0 & $\mathbf{0}$ & $\mathbf{0}$ & $\mathbf{0}$ & $\mathbf{0}$ \\
\hline bah13an & 1 & 3 & 2 & 1 & 3 & 2 & hjh07an & 2 & 3 & 3 & 2 & 3 & 3 & ljs31an & 2 & 1 & 1 & 1 & 1 & 0 & rhp12an & 2 & 3 & 2 & 2 & 3 & 2 \\
\hline bef05an & 2 & 3 & 3 & 2 & 0 & 0 & hlm24an & 1 & 2 & 2 & 1 & 2 & 2 & llalnal & 1 & 0 & 0 & 0 & 0 & 0 & rjf22an & 2 & 3 & 3 & 2 & 3 & 3 \\
\hline bjblnal & 0 & $\mathbf{0}$ & 0 & $\mathbf{0}$ & $\mathbf{0}$ & 0 & hxi29an & 1 & 3 & 2 & 1 & 3 & 2 & $1 \operatorname{lm} 22 \mathrm{an}$ & 3 & 3 & 2 & 3 & 3 & 2 & rjl28an & 3 & 3 & 2 & 2 & 3 & 2 \\
\hline bjvlnal & 0 & $\mathbf{0}$ & 0 & 0 & 0 & 0 & hxl58an & 1 & 0 & 0 & 1 & 0 & 0 & lmvlnal & 1 & 0 & 0 & 1 & 0 & 0 & rjr15an & 1 & 1 & 1 & 1 & 1 & 1 \\
\hline bkb13an & 1 & 0 & 1 & 2 & 0 & 1 & jaflnal & 0 & 0 & 0 & 0 & 0 & 0 & lmwlnal & 1 & 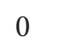 & 0 & . & 0 & 0 & rjslnal & 0 & 0 & 0 & 0 & 0 & 0 \\
\hline blb03an & 2 & 3 & 2 & 2 & 3 & 2 & janlnal & 1 & 1 & 1 & 1 & 1 & 1 & lncllan & 1 & 0 & 0 & $\mathbf{0}$ & 0 & 0 & rjz16an & 1 & 0 & 0 & 1 & 0 & 0 \\
\hline bpf03an & 1 & 2 & 1 & 1 & 2 & 1 & jap02an & 2 & 1 & 1 & 2 & 0 & 1 & lrd21an & 1 & 0 & 0 & 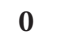 & 0 & 0 & rmb07an & 2 & 3 & 3 & 2 & 3 & 3 \\
\hline bsg13an & 1 & 1 & 1 & 1 & 1 & 1 & japlnal & 0 & 0 & 0 & 0 & 0 & 0 & $\operatorname{lvd} 28 \mathrm{a}$ & 2 & 3 & 2 & 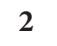 & 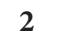 & 2 & rpj15an & 3 & 3 & 3 & 3 & 3 & 3 \\
\hline cacl0an & 2 & 3 & 3 & 2 & 0 & 0 & jccl0an & 2 & 2 & 2 & 2 & 2 & 2 & lwr18an & 1 & 3 & 3 & 1 & 0 & 0 & rpq20an & 2 & 3 & 3 & 2 & 3 & 3 \\
\hline cadlnal & 0 & $\mathbf{0}$ & $\mathbf{0}$ & $\mathbf{0}$ & $\mathbf{0}$ & $\mathbf{0}$ & jcr0lan & 3 & 3 & 3 & 3 & 3 & 3 & lxc01an & 2 & 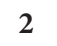 & 2 & 2 & 0 & 0 & rtllian & 1 & 0 & 1 & 1 & 0 & 1 \\
\hline cak25an & 2 & 1 & 1 & 2 & 1 & 1 & jeglnal & 0 & $\mathbf{0}$ & 0 & $\mathbf{0}$ & $\mathbf{0}$ & 0 & lxc06an & 3 & 3 & 3 & 3 & 3 & 3 & rwc23an & 2 & 3 & 3 & 2 & 3 & 3 \\
\hline ceblnal & 0 & $\mathbf{0}$ & 0 & 0 & $\mathbf{0}$ & $\mathbf{0}$ & jeg29an & 2 & 3 & 2 & 2 & 0 & 0 & lxr15an & 3 & 3 & 2 & 3 & 3 & 2 & rxm15an & 1 & 0 & 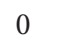 & 1 & 0 & 0 \\
\hline cls31an & 2 & 1 & 1 & 2 & 1 & 1 & jfg08an & 3 & 3 & 3 & 3 & 3 & 3 & mab06an & 2 & 1 & 1 & 2 & 1 & 1 & rxp02an & 1 & 3 & 3 & 1 & 3 & 3 \\
\hline cma06an & 1 & 2 & 1 & 1 & 2 & 1 & jfn2lan & 3 & 2 & 2 & 3 & 2 & 2 & $\operatorname{mam} 08 a$ & 2 & 3 & 3 & 2 & 3 & 3 & sacl0an & 2 & 3 & 3 & 2 & 3 & 3 \\
\hline cmr06an & 0 & 0 & 0 & 0 & $\mathbf{0}$ & 0 & jhw29an & 2 & 1 & 1 & 2 & 1 & 1 & mamln & 0 & $\mathbf{0}$ & 0 & 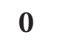 & 0 & $\mathbf{0}$ & sae0lan & 1 & 0 & 0 & 1 & 0 & 0 \\
\hline crml & 3 & 3 & 3 & 3 & 2 & 2 & jkrlnal & 1 & 0 & 0 & 1 & 0 & 0 & masln & 0 & 8 & 0 & 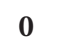 & 0 & 0 & sav18an & 3 & 3 & 3 & 3 & 3 & 3 \\
\hline ctb30an & 2 & 2 & 1 & 2 & 1 & 1 & jld24an & 2 & 3 & 2 & 2 & 3 & 2 & mcblnal & 1 & 0 & 0 & 1 & 0 & 0 & sbf1lan & 0 & 0 & 0 & 0 & 0 & 0 \\
\hline dajlnal & 0 & $\mathbf{0}$ & $\mathbf{0}$ & 0 & $\mathbf{0}$ & $\mathbf{0}$ & jls1lan & 2 & 1 & 1 & 2 & 1 & 1 & mcw21an & 2 & 1 & 1 & 2 & 1 & 1 & scc15an & 3 & 3 & 3 & 3 & 0 & 1 \\
\hline $\mathrm{d}$ & 2 & 2 & 1 & 2 & 2 & 1 & jmcl8an & 1 & 0 & 0 & 1 & 0 & 0 & mec & 2 & 0 & 0 & 2 & 0 & 0 & scklnal & 1 & 0 & 0 & 1 & 0 & 0 \\
\hline das30an & 1 & - & 1 & 1 & - & 1 & jmclnal & 1 & 0 & 0 & 1 & 0 & 0 & mec28an & 2 & 2 & 1 & 1 & 2 & 1 & sctlnal & 1 & 0 & 0 & 1 & 0 & 0 \\
\hline $\mathrm{dbfl} 18$ & 2 & 2 & 1 & 2 & 2 & 1 & jpp27an & 3 & 3 & 3 & 3 & 0 & 0 & $\mathrm{mfc}$ & 3 & 3 & 3 & 2 & 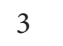 & 3 & 1 & 1 & 0 & 0 & 1 & 0 & 0 \\
\hline dfplnal & 0 & $\mathbf{0}$ & $\mathbf{0}$ & 0 & 0 & $\mathbf{0}$ & jrf30an & 1 & 0 & 0 & 1 & 0 & 0 & $\mathrm{mfml}$ & 1 & 0 & 0 & 1 & 0 & 0 & sec02an & 2 & 0 & 1 & 2 & 0 & 1 \\
\hline djglnal & 0 & $\mathbf{0}$ & 0 & 0 & $\mathbf{0}$ & 0 & jthlnal & 1 & 0 & 0 & $\mathbf{0}$ & $\mathbf{0}$ & $\mathbf{0}$ & mjuln & 0 & , & $\mathbf{0}$ & 0 & 0 & 0 & sef10an & 1 & 0 & 0 & 1 & 0 & 0 \\
\hline djp04an & 2 & 2 & 2 & 2 & 2 & 2 & jtm05an & 1 & 2 & 1 & 1 & 2 & 1 & mpb23an & 3 & 3 & 3 & 3 & 3 & 3 & seg18an & 2 & 1 & 1 & 2 & 1 & 1 \\
\hline dmalnal & 0 & 0 & 0 & 0 & 0 & 0 & jxclnal & 1 & 0 & 0 & 0 & 0 & 0 & $\mathrm{mpf} 25 \mathrm{a}$ & 1 & 2 & 1 & 1 & 2 & 1 & sek06an & 2 & 2 & 1 & 2 & 2 & 1 \\
\hline $\mathrm{dmc}$ & 2 & 3 & 2 & 2 & 0 & 0 & jxc2lan & 2 & 0 & 0 & 2 & 0 & 0 & $\mathrm{mps}$ & 2 & 0 & 1 & 2 & 0 & 1 & shd04an & 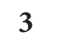 & 3 & 3 & 3 & 3 & 3 \\
\hline $\mathrm{dmp}$ & 2 & 2 & 2 & 2 & 2 & 2 & jxd30an & 1 & 1 & 1 & 0 & 1 & 1 & mrblla & 1 & 3 & 2 & 1 & 3 & 2 & sislnal & 0 & 0 & 0 & 0 & 0 & 0 \\
\hline drcl & 2 & 2 & 2 & 2 & 0 & 0 & jxf1lan & 3 & 3 & 3 & 3 & 3 & 3 & $\operatorname{mrc} 20$ & 2 & 3 & 2 & 2 & 3 & 2 & sjd28an & 1 & 1 & 1 & 1 & 1 & 1 \\
\hline dsc25an & 3 & 3 & 3 & 3 & 0 & 0 & kab03an & 1 & 0 & 0 & 1 & 0 & 0 & mwd28an & 2 & 3 & 2 & 2 & 3 & 2 & slclnal & 2 & 0 & 0 & 1 & 0 & 0 \\
\hline 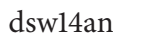 & 2 & - & 1 & 2 & - & 1 & & 1 & 0 & 0 & 1 & 0 & 0 & & 0 & & 0 & & 0 & 0 & & 2 & 0 & 1 & 1 & 0 & 1 \\
\hline dvd & 3 & 3 & 3 & 3 & 3 & 3 & kanl & 0 & 0 & 0 & 0 & 0 & 0 & $\mathrm{mxc10a}$ & 2 & 2 & 2 & 2 & 2 & 2 & slg05an & 1 & 0 & 0 & 1 & 0 & 0 \\
\hline dwk04an & 2 & 2 & 2 & 2 & 2 & 2 & kcg23an & 2 & 2 & 1 & 2 & 2 & 1 & $\operatorname{mxn} 24 a n$ & 1 & 0 & 0 & 1 & 0 & 0 & sma08an & 3 & 3 & 3 & 3 & 3 & 3 \\
\hline dws & 0 & $\mathbf{0}$ & $\mathbf{0}$ & 0 & $\mathbf{0}$ & $\mathbf{0}$ & cg25an & 1 & 0 & 0 & 1 & 0 & 0 & $\mathrm{~m} s$ & 1 & 0 & 0 & $\mathbf{0}$ & $\mathbf{0}$ & 0 & sws04an & 3 & 2 & 2 & 3 & 2 & 2 \\
\hline eab27an & 3 & 3 & 3 & 3 & 3 & 3 & kdb23an & 2 & 2 & 2 & 2 & 2 & 2 & nfg08an & 2 & 2 & 1 & 1 & 2 & 1 & sxvlnal & 1 & 0 & 0 & 1 & 0 & 0 \\
\hline easllan & 1 & 2 & 1 & 1 & 2 & 1 & kjb19an & 2 & 3 & 3 & 2 & 3 & 3 & njs06a & 2 & 2 & 1 & 2 & 2 & 1 & tab2 & 3 & 3 & 3 & 3 & 3 & 3 \\
\hline eas15an & 3 & 3 & 3 & 3 & 3 & 3 & klc06an & 2 & 3 & 3 & 2 & 0 & 0 & njslnal & 1 & 0 & 0 & 1 & 0 & 0 & tdh12an & 2 & 2 & 1 & 2 & 2 & 1 \\
\hline edclnal & 1 & 0 & 0 & 1 & 0 & 0 & klc09an & 2 & 3 & 2 & 2 & 3 & 2 & nkr03a & 1 & 0 & 0 & 1 & 0 & 0 & tlp13an & 2 & 2 & 2 & 2 & 2 & 2 \\
\hline eec04an & 3 & 3 & 3 & 3 & 2 & 2 & kld26an & 2 & 2 & 1 & 2 & 2 & 1 & nlc08a & 2 & 2 & 1 & 2 & 2 & 1 & tls09an & 2 & 2 & 2 & 2 & 2 & 2 \\
\hline eed07an & 3 & 3 & 3 & 3 & 3 & 3 & kmc22an & 2 & 1 & 1 & 2 & 1 & 0 & nmb28an & 2 & 3 & 2 & 1 & 3 & 2 & tpp24an & 1 & 0 & 0 & 1 & 0 & 0 \\
\hline ejclnal & 0 & $\mathbf{0}$ & 0 & 0 & $\mathbf{0}$ & 0 & kms29an & 2 & 3 & 3 & 2 & 3 & 3 & nmc22an & 2 & 2 & 1 & 1 & 1 & 1 & tps16an & 1 & - & 1 & 1 & - & 1 \\
\hline ejh24an & 3 & 3 & 3 & 3 & 0 & 1 & kmw05an & 3 & 3 & 3 & 3 & 1 & 1 & nml15an & 0 & 1 & 1 & 0 & 1 & 1 & txnlnal & 1 & - & 0 & 1 & - & 0 \\
\hline emp27an & 2 & 2 & 2 & 2 & 2 & 2 & kps25an & 0 & 2 & 1 & 0 & 2 & 1 & nmv07an & 3 & 3 & 3 & 3 & 0 & 0 & vaw07an & 3 & 3 & 3 & 3 & 3 & 3 \\
\hline ess05an & 1 & 0 & 0 & 1 & 0 & 0 & ktj26an & 3 & 3 & 3 & 3 & 3 & 3 & oab28an & 1 & 3 & 3 & 2 & 3 & 3 & vmclnal & 1 & 0 & 0 & 1 & 0 & 0 \\
\hline eww05an & 2 & 2 & 2 & 2 & 2 & 2 & kxb17an & 3 & 3 & 3 & 3 & 3 & 3 & ovklnal & 0 & 0 & $\mathbf{0}$ & $\mathbf{0}$ & 0 & 0 & wcb24an & 1 & 0 & 0 & 1 & 0 & 0 \\
\hline fmblnal & 0 & 0 & 0 & 0 & 0 & 0 & kxh30an & 3 & 3 & 3 & 3 & 3 & 3 & pat10an & 2 & 3 & 3 & 1 & 3 & 3 & wdk1nal & $\mathbf{0}$ & 0 & $\mathbf{0}$ & 0 & 0 & 0 \\
\hline fmrl7an & 3 & 3 & 3 & 3 & 3 & 3 & lac02an & 2 & 1 & 1 & 2 & 0 & 1 & pbdlnal & 1 & 0 & 0 & 1 & 0 & 0 & wfc07an & 2 & 3 & 2 & 2 & 3 & 2 \\
\hline fxc12an & 1 & 2 & 2 & 1 & 2 & 2 & lad13an & 1 & 2 & 1 & 1 & 2 & 1 & pcalnal & 1 & 0 & 0 & 0 & 0 & 0 & wjb06an & 2 & 0 & 0 & 2 & 0 & 0 \\
\hline gdr15an & 3 & 3 & 3 & 3 & 0 & 0 & ladlnal & 0 & 0 & 0 & 0 & 0 & 0 & pdollan & 2 & 3 & 3 & 2 & 3 & 3 & wjp20an & 3 & 3 & 3 & 3 & 3 & 3 \\
\hline gmm09an & 3 & 3 & 3 & 3 & 3 & 3 & lai04an & 1 & 3 & 2 & 1 & 3 & 2 & pgb16an & 1 & 1 & 1 & 1 & 1 & 1 & wpb30an & 2 & 1 & 1 & 2 & 1 & 1 \\
\hline gms05an & 2 & 3 & 3 & 2 & 3 & 3 & lap05an & 1 & 3 & 3 & 1 & 3 & 3 & plw14an & 2 & 2 & 2 & 2 & 2 & 2 & wxe04an & 3 & 3 & 3 & 3 & 3 & \\
\hline
\end{tabular}


Despite the promising results, an accurate comparison with the studies found in the state of the art is difficult since, as stated in [65], different works tend to use different types of corpus and methodologies, and results are unfortunately dependant of the corpus used for training and validation. Furthermore, those cases on which different studies utilize the same database, labeling is usually different. In this sense, the definition of a standardized database with a consistent and known labeling would lead to comparable results. For this reason, with the aim of providing the scientific community with a benchmark labeling and to promote a more solid comparative estimate of future techniques and studies, the labeling of the $G$ and $R$ features used on this work has been included in Table 6. Despite its known limitations [65], the fact that MEEI database is commercially available for researchers is also an advantage in this sense.

On the other hand, other approaches such as [11,23] have already demonstrated that MS is a good source of information to detect pathological voices or to perform an automatic pathology classification. The main difference with respect to Markaki's approach is that in this study MS is used to evaluate the speech according to a 4-level scale in two different features of the speech: Grade and Roughness. On the other hand, the parameters used in the present study are less abstract and have an easier physical interpretation, opening the possibility of using them in a clinical setting.

In spite of the good figures, MS has a weakness which could make it a nonviable parameterization in some applications: computational cost. Depending on the configuration and frequency margins, to calculate a MS matrix can take around 400 times more than to calculate MFCCs on the same signal frame.

Regarding the future work, all MS parameters must be studied and adjusted separately to find the adequate frequency margins of operation to optimize results. In addition, the use of the proposed MS Morphological Parameters in combination with some other features such as complexity and noise measurements or cepstral-based coefficients to characterize GRBAS traits would be advisable. Moreover, the study of regression methods like Support Vector Regression [66] and other feature selection techniques such as Least Absolute Shrinkage and Selection Operator (LASSO) [67] is of interest. In respect of the classification stage, the stratification of the speakers according to her/his sex, age, or emotional state could increase performance as suggested in [68]. For this purpose, a priori categorization of speaker's characteristics using hierarchical methods might be used to simplify the statistical models behind to automatically assess the quality of speech.

Summarizing, results suggest that the proposed MS Morphological Parameters are an objective basis to help clinicians to assess Grade and Roughness according the GRBAS scale, reducing uncertainty and making the assessment easier to replicate. It would be advisable to study the synthesis of a new parameter combining the proposed MS Morphological Parameters, being suitable for therapists and physicians. In view of the experiments carried out in this work, there are evidences that suggest that the use of these parameters provides better results than the classic MFCCs, traditionally used to characterize voice signals. On the other hand, its main drawback is the initial difficulty of applying the proposed MSbased parameters to the study of running speech.

\section{Appendix}

On Table 6 perceptual assessment of GRBAS Grade and Roughness traits for 224 recordings of MEEI corpus [55] can be found. $G 1$ and $R 1$ are assessments of Therapist 1 . The rest of evaluations are performed by Therapist 2 in two different sessions. Numbers in bold represent the agreement subsets while all evaluations are the original subsets.

\section{Conflict of Interests}

The authors declare that there is no conflict of interests regarding the publication of this paper.

\section{Acknowledgments}

The authors of this paper have developed their work under the grant of the project TEC2012-38630-C04-01 from the Ministry of Economy and Competitiveness of Spain and Ayudas para la realizacion del doctorado (RR01/2011) from Universidad Politecnica de Madrid, Spain.

\section{References}

[1] C. Sapienza and B. Hoffman-Ruddy, Voice Disorders, Plural Publishing, 2009.

[2] D. K. Wilson, Voice Problems of Children, Williams \& Wilkins, Baltimore, Md, USA, 1987.

[3] G. B. Kempster, B. R. Gerratt, K. V. Abbott, J. BarkmeierKraemer, and R. E. Hillman, "Consensus auditory-perceptual evaluation of voice: development of a standardized clinical protocol," American Journal of Speech-Language Pathology, vol. 18, no. 2, pp. 124-132, 2009.

[4] M. Hirano, Clinical Examination of Voice, Springer, 1981.

[5] M. S. De Bodt, F. L. Wuyts, P. H. van de Heyning, and C. Croux, "Test-retest study of the GRBAS scale: influence of experience and professional background on perceptual rating of voice quality," Journal of Voice, vol. 11, no. 1, pp. 74-80, 1997.

[6] I. V. Bele, "Reliability in perceptual analysis of voice quality," Journal of Voice, vol. 19, no. 4, pp. 555-573, 2005.

[7] A. Tsanas, M. A. Little, P. E. McSharry, and L. O. Ramig, "Accurate telemonitoring of Parkinson's disease progression by noninvasive speech tests," IEEE Transactions on Biomedical Engineering, vol. 57, no. 4, pp. 884-893, 2010.

[8] A. Tsanas, M. A. Little, C. Fox, and L. O. Ramig, "Objective automatic assessment of rehabilitative speech treatment in parkinson's disease," IEEE Transactions on Neural Systems and Rehabilitation Engineering, vol. 22, no. 1, pp. 181-190, 2014.

[9] C. Fredouille, G. Pouchoulin, A. Ghio, J. Revis, J.-F. Bonastre, and A. Giovanni, "Back-and-forth methodology for objective voice quality assessment: from/to expert knowledge to/from automatic classification of dysphonia," EURASIP Journal on Advances in Signal Processing, vol. 2009, Article ID 982102, 13 pages, 2009.

[10] P. Gómez-Vilda, R. Fernández-Baillo, V. Rodellar-Biarge et al., "Glottal Source biometrical signature for voice pathology 
detection," Speech Communication, vol. 51, no. 9, pp. 759-781, 2009.

[11] M. Markaki and Y. Stylianou, "Voice pathology detection and discrimination based on modulation spectral features," IEEE Transactions on Audio, Speech and Language Processing, vol. 19, no. 7, pp. 1938-1948, 2011.

[12] J. D. Arias-Londoño, J. I. Godino-Llorente, M. Markaki, and Y. Stylianou, "On combining information from modulation spectra and mel-frequency cepstral coefficients for automatic detection of pathological voices," Logopedics Phoniatrics Vocology, vol. 36, no. 2, pp. 60-69, 2011.

[13] R. Wielgat, T. P. Zieliński, T. Woźniak, S. Grabias, and D. Król, "Automatic recognition of pathological phoneme production," Folia Phoniatrica et Logopaedica, vol. 60, no. 6, pp. 323-331, 2009.

[14] L. Salhi and A. Cherif, "Robustness of auditory teager energy cepstrum coefficients for classification of pathological and normal voices in noisy environments," The Scientific World Journal, vol. 2013, Article ID 435729, 8 pages, 2013.

[15] M. Rosa, J. Pereira, M. Greller, and A. Carvalho, "Signal processing and statistical procedures to identify laryngeal pathologies," in Proceedings of the 6th IEEE International Conference on Electronics, Circuits and Systems (ICECS '99), vol. 1, pp. 423-426, Pafos, Cyprus, 1999.

[16] G. Muhammad, M. Alsulaiman, A. Mahmood, and Z. Ali, "Automatic voice disorder classification using vowel formants," in Proceedings of the 12th IEEE International Conference on Multimedia and Expo (ICME '11), pp. 1-6, IEEE, July 2011.

[17] P. Yu, Z. Wang, S. Liu, N. Yan, L. Wang, and M. Ng, "Multidimensional acoustic analysis for voice quality assessment based on the GRBAS scale," in Proceedings of the 9th International Symposium on Chinese Spoken Language Processing (ISCSLP '14), pp. 321-325, IEEE, Singapore, September 2014.

[18] A. Tsanas, M. A. Little, P. E. McSharry, and L. O. Ramig, "Nonlinear speech analysis algorithms mapped to a standard metric achieve clinically useful quantification of average Parkinson's disease symptom severity," Journal of the Royal Society Interface, vol. 8, no. 59, pp. 842-855, 2011.

[19] G. Pouchoulin, C. Fredouille, J.-F. Bonastre, A. Ghio, and A. Giovanni, "Frequency study for the characterization of the dysphonic voices," in Proceedings of the 8th Annual Conference of the International Speech Communication Association (Interspeech '07), pp. 1198-1201, Antwerp, Belgium, August 2007.

[20] G. Pouchoulin, C. Fredouille, J. Bonastre et al., "Dysphonic voices and the $0-3000 \mathrm{~Hz}$ frequency band," in Proceedings of the 9th Annual Conference of the International Speech Communication Association (Interspeech '08), pp. 2214-2217, ISCA, Brisbane, Australia, September 2008.

[21] N. Sáenz-Lechón, J. I. Godino-Llorente, V. Osma-Ruiz, M. Blanco-Velasco, and F. Cruz-Roldán, "Automatic assessment of voice quality according to the GRBAS scale," in Proceedings of the 28th Annual International Conference of the IEEE Engineering in Medicine and Biology Society (EMBS '06), pp. 2478-2481, September 2006.

[22] A. Stráník, R. Čmejla, and J. Vokřál, “Acoustic parameters for classification of breathiness in continuous speech according to the GRBAS scale," Journal of Voice, vol. 28, no. 5, pp. 653.e9653.e17, 2014.

[23] M. Markaki and Y. Stylianou, "Modulation spectral features for objective voice quality assessment: the breathiness case," in Proceedings of the 6th International Workshop on Models and
Analysis of Vocal Emissions for Biomedical Applications, Firenze, Italy, December 2009.

[24] W. S. Winholtz and L. O. Ramig, "Vocal tremor analysis with the vocal demodulator," Journal of Speech and Hearing Research, vol. 35, no. 3, pp. 562-573, 1992.

[25] M. A. Little, P. E. McSharry, S. J. Roberts, D. A. E. Costello, and I. M. Moroz, "Exploiting nonlinear recurrence and fractal scaling properties for voice disorder detection," BioMedical Engineering Online, vol. 6, article 23, 2007.

[26] C. Peng, W. Chen, X. Zhu, B. Wan, and D. Wei, "Pathological voice classification based on a single Vowel's acoustic features," in Proceedings of the 7th IEEE International Conference on Computer and Information Technology (CIT '07), pp. 1106-1110, October 2007.

[27] J. I. Godino-Llorente and P. Gómez-Vilda, "Automatic detection of voice impairments by means of short-term cepstral parameters and neural network based detectors," IEEE Transactions on Biomedical Engineering, vol. 51, no. 2, pp. 380-384, 2004.

[28] C. Maguire, P. de Chazal, R. B. Reilly, and P. D. Lacy, "Identification of voice pathology using automated speech analysis," in Proceedings of the 3rd International Workshop on Models and Analysis of Vocal Emissions for Biomedical Applications (MAVEBA '03), pp. 259-262, Florence, Italy, December 2003.

[29] M. Marinaki and C. Kotropoulos, "Automatic detection of vocal fold paralysis and edema," in Proceedings of the ICSLP, Jeju Island, Republic of Korea, 2004.

[30] J. Godino-Llorente, P. Gómez-Vilda, N. Sáenz-Lechón, M. Blanco-Velasco, F. Cruz-Roldán, and M. A. Ferrer, "Discriminative methods for the detection of voice disorders," in Proceedings of the International Conference on Non-Linear Speech Processing (NOLISP '05), pp. 158-167, Barcelona, Spain, April 2005.

[31] K. Shama, A. Krishna, and N. U. Cholayya, "Study of harmonics-to-noise ratio and critical-band energy spectrum of speech as acoustic indicators of laryngeal and voice pathology," EURASIP Journal on Advances in Signal Processing, vol. 2007, no. 1, Article ID 85286, 2007.

[32] A. I. Fontes, P. T. Souza, A. D. Neto, A. d. Martins, and L. F. Silveira, "Classification system of pathological voices using correntropy," Mathematical Problems in Engineering, vol. 2014, Article ID 924786, 7 pages, 2014.

[33] R. Kohavi, "A study of cross-validation and bootstrap for accuracy estimation and model selection," in Proceedings of the 14th International Joint Conference on Artificial Intelligence (IJCAI '95), vol. 2, pp. 1137-1145, 1995.

[34] S. Jannetts and A. Lowit, "Cepstral analysis of hypokinetic and ataxic voices: correlations with perceptual and other acoustic measures," Journal of Voice, vol. 28, no. 6, pp. 673-680, 2014.

[35] P. Boersma, "Praat, a system for doing phonetics by computer," Glot International, vol. 5, no. 9-10, pp. 341-345, 2002.

[36] J. D. Arias-Londoño, J. I. Godino-Llorente, N. Sáenz-Lechón et al., "Automatic GRBAS assessment using complexity measures and a multiclass GMM-based detector," in Proceedings of the 7th International Workshop on Models and Analysis of Vocal Emissions for Biomedical Applications, 2011.

[37] N. C. Singha and F. E. Theunissen, "Modulation spectra of natural sounds and ethological theories of auditory processing," The Journal of the Acoustical Society of America, vol. 114, no. 6, pp. 3394-3411, 2003.

[38] L. Atlas and S. A. Shamma, "Joint acoustic and modulation frequency," EURASIP Journal on Applied Signal Processing, vol. 2003, no. 7, pp. 668-675, 2003. 
[39] S.-C. Lim, S.-J. Jang, S.-P. Lee, and M. Y. Kim, "Music genre/mood classification using a feature-based modulation spectrum," in Proceedings of the International Conference on Mobile IT-Convergence (ICMIC '11), pp. 133-136, IEEE, September 2011.

[40] W.-Y. Chu, J.-W. Hung, and B. Chen, "Modulation spectrum factorization for robust speech recognition," in Proceedings of the Asia-Pacific Signal and Information Processing Association Annual Summit and Conference (APSIPA ASC '11), pp. 1-6, October 2011.

[41] H.-T. Fan, Y.-C. Tsai, and J.-W. Hung, "Enhancing the sub-band modulation spectra of speech features via nonnegative matrix factorization for robust speech recognition," in Proceedings of the International Conference on System Science and Engineering (ICSSE '12), pp. 179-182, July 2012.

[42] E. Bozkurt, O. Toledo-Ronen, A. Sorin, and R. Hoory, "Exploring modulation spectrum features for speech-based depression level classification," in Proceedings of the 15th Annual Conference of the International Speech Communication Association, Singapore, September 2014.

[43] K. M. Carbonell, R. A. Lester, B. H. Story, and A. J. Lotto, "Discriminating simulated vocal tremor source using amplitude modulation spectra," Journal of Voice, vol. 29, no. 2, pp. 140-147, 2015.

[44] P. H. Dejonckere, C. Obbens, G. M. de Moor, and G. H. Wieneke, "Perceptual evaluation of dysphonia: reliability and relevance," Folia Phoniatrica, vol. 45, no. 2, pp. 76-83, 1993.

[45] M. P. Karnell, S. D. Melton, J. M. Childes, T. C. Coleman, S. A. Dailey, and H. T. Hoffman, "Reliability of clinician-based (grbas and cape-v) and patientbased (v-rqol and ipvi) documentation of voice disorders," Journal of Voice, vol. 21, no. 5, pp. 576-590, 2007.

[46] L. Rabiner and B.-H. Juang, Fundamentals of Speech Recognition, Prentice Hall, New York, NY, USA, 1993.

[47] S. M. Schimmel, L. E. Atlas, and K. Nie, "Feasibility of single channel speaker separation based on modulation frequency analysis," in Proceedings of the IEEE International Conference on Acoustics, Speech and Signal Processing (ICASSP '07), vol. 4, pp. IV605-IV608, April 2007.

[48] L. Atlas, P. Clark, and S. Schimmel, "Modulation Toolbox Version 2.1 for MATLAB," 2010, http://isdl.ee.washington.edu/ projects/modulationtoolbox/.

[49] S. M. Schimmel and L. E. Atlas, "Coherent envelope detection for modulation filtering of speech," in Proceedings of the IEEE International Conference on Acoustics, Speech, and Signal Processing (ICASSP '05), vol. 1, pp. I221-I224, IEEE, March 2005.

[50] R. Cusack and N. Papadakis, "New robust 3-D phase unwrapping algorithms: application to magnetic field mapping and undistorting echoplanar images," NeuroImage, vol. 16, no. 3, pp. 754-764, 2002.

[51] B. Gajić and K. K. Paliwal, "Robust speech recognition in noisy environments based on subband spectral centroid histograms," IEEE Transactions on Audio, Speech and Language Processing, vol. 14, no. 2, pp. 600-608, 2006.

[52] R. J. Elble, "Central mechanisms of tremor," Journal of Clinical Neurophysiology, vol. 13, no. 2, pp. 133-144, 1996.

[53] M. Bové, N. Daamen, C. Rosen, C.-C. Wang, L. Sulica, and J. Gartner-Schmidt, "Development and validation of the vocal tremor scoring system," The Laryngoscope, vol. 116, no. 9, pp. 1662-1667, 2006.

[54] R. Peters and R. Strickland, "Image complexity metrics for automatic target recognizers," in Proceedings of the Automatic
Target Recognizer System and Technology Conference, October 1990.

[55] Voice Disorders Database, Kay Elemetrics Corporation, Lincoln Park, NJ, USA, 1994.

[56] V. Parsa and D. G. Jamieson, "Identification of pathological voices using glottal noise measures," Journal of Speech, Language, and Hearing Research, vol. 43, no. 2, pp. 469-485, 2000.

[57] L. Smith, A Tutorial on Principal Components Analysis, vol. 51, Cornell University, Ithaca, NY, USA, 2002.

[58] R. Haeb-Umbach and H. Ney, "Linear discriminant analysis for improved large vocabulary continuous speech recognition," in Proceedings of the IEEE International Conference on Acoustics, Speech, and Signal Processing (ICASSP '92), vol. 1, pp. 13-16, San Francisco, Calif, USA, March 1992.

[59] B. Efron and G. Gong, "A leisurely look at the bootstrap, the jackknife, and cross-validation," The American Statistician, vol. 37, no. 1, pp. 36-48, 1983.

[60] T. K. Moon, “The expectation-maximization algorithm," IEEE Signal Processing Magazine, vol. 13, no. 6, pp. 47-60, 1996.

[61] J. Cohen, "A coefficient of agreement for nominal scales," Educational and Psychological Measurement, vol. 20, no. 1, pp. 37-46, 1960.

[62] D. G. Altman, Practical Statistics for Medical Research, CRC Press, 1990.

[63] H. He and E. A. Garcia, "Learning from imbalanced data," IEEE Transactions on Knowledge and Data Engineering, vol. 21, no. 9, pp. 1263-1284, 2009.

[64] D. G. Childers, "Detection of laryngeal function using speech and electroglottographic data," IEEE Transactions on Biomedical Engineering, vol. 39, no. 1, pp. 19-25, 1992.

[65] N. Sáenz-Lechón, J. I. Godino-Llorente, V. Osma-Ruiz, and P. Gómez-Vilda, "Methodological issues in the development of automatic systems for voice pathology detection," Biomedical Signal Processing and Control, vol. 1, no. 2, pp. 120-128, 2006.

[66] V. N. Vapnik and V. Vapnik, Statistical Learning Theory, vol. 2 of Adaptive and Learning Systems for Signal Processing, Communications, and Control, John Wiley \& Sons, 1998.

[67] R. Tibshirani, "Regression shrinkage and selection via the lasso," Journal of the Royal Statistical Society Series B: Methodological, vol. 58, no. 1, pp. 267-288, 1996.

[68] R. Fraile, N. Sáenz-Lechón, J. I. Godino-Llorente, V. OsmaRuiz, and C. Fredouille, "Automatic detection of laryngeal pathologies in records of sustained vowels by means of melfrequency cepstral coefficient parameters and differentiation of patients by sex," Folia Phoniatrica et Logopaedica, vol. 61, no. 3, pp. 146-152, 2009. 


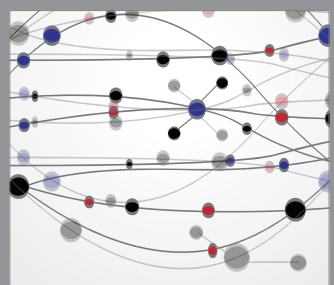

The Scientific World Journal
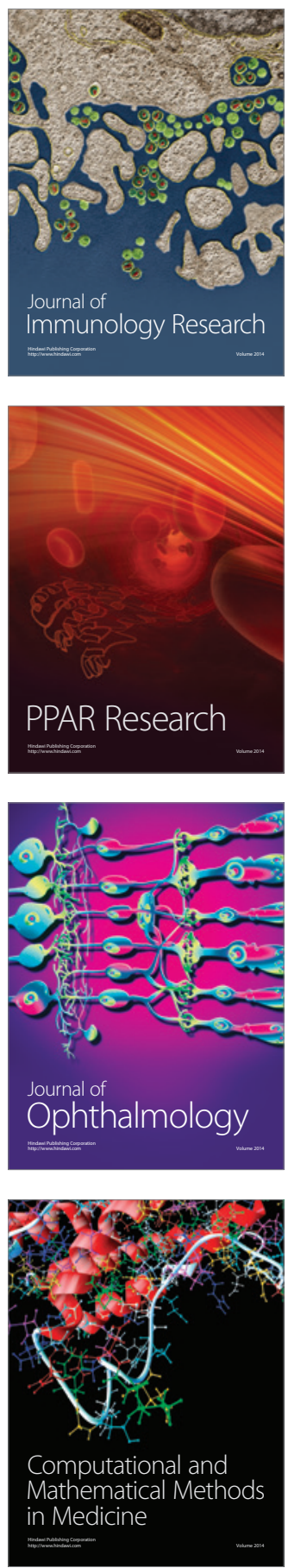

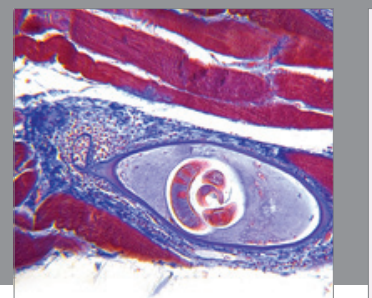

Gastroenterology

Research and Practice
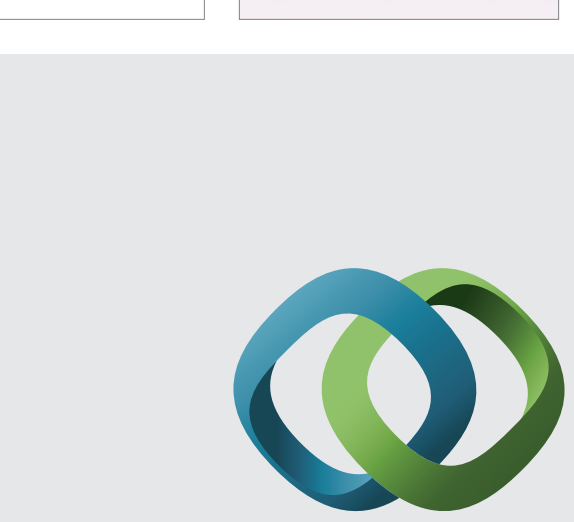

\section{Hindawi}

Submit your manuscripts at

http://www.hindawi.com
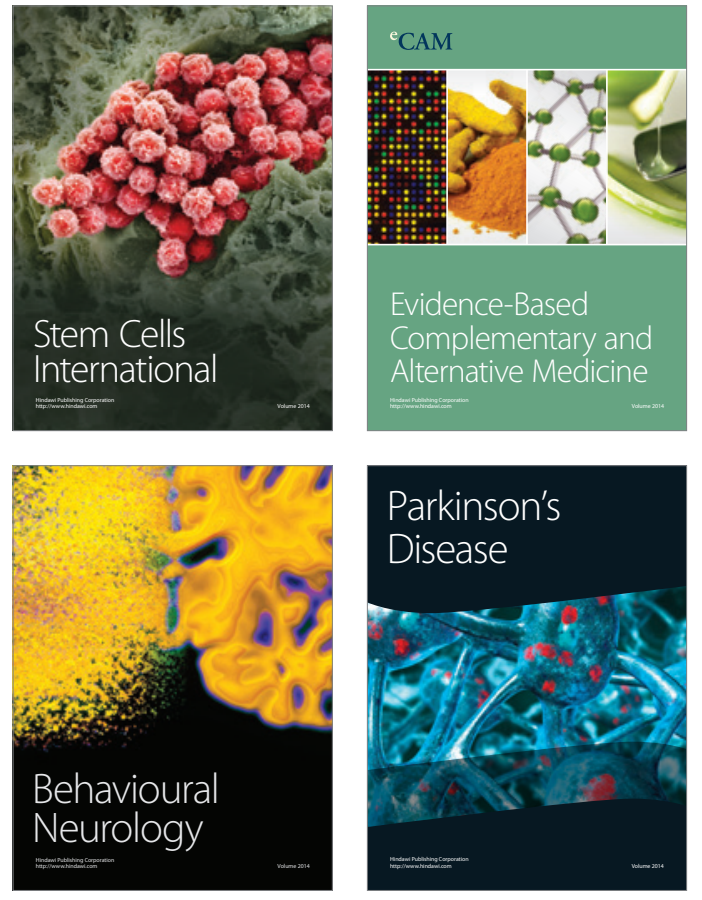
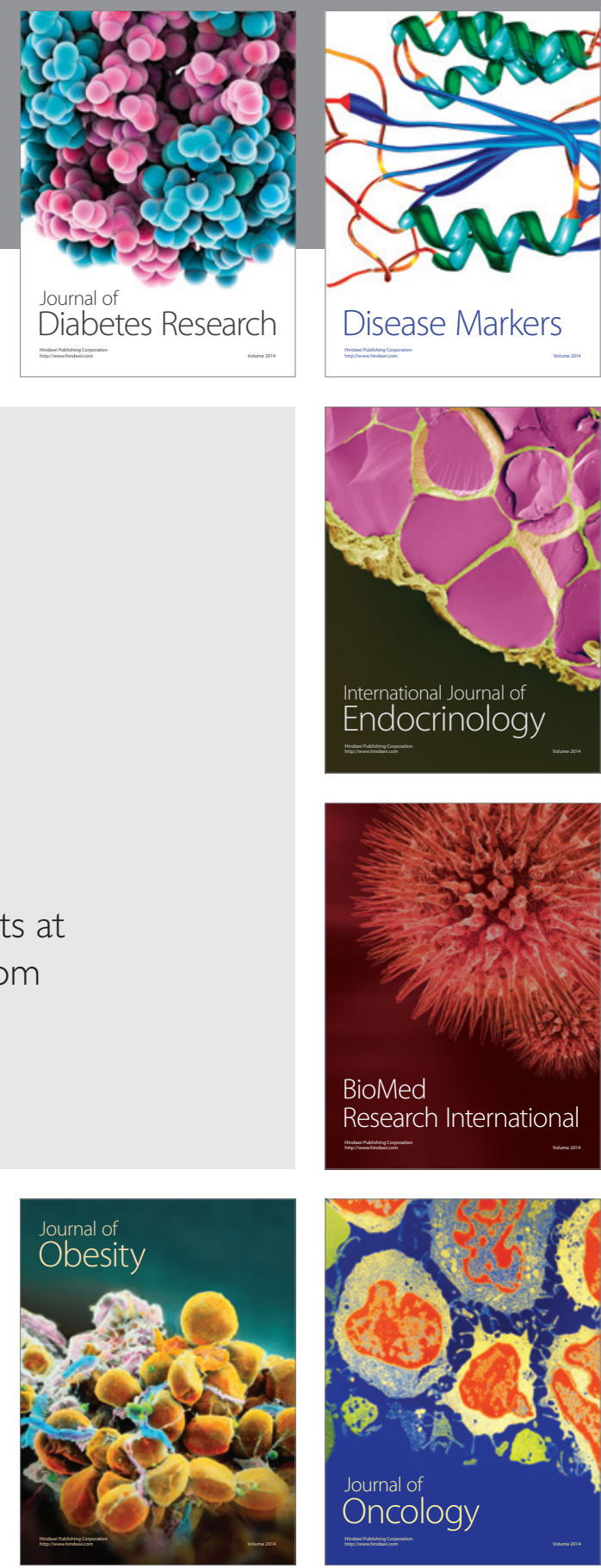

Disease Markers
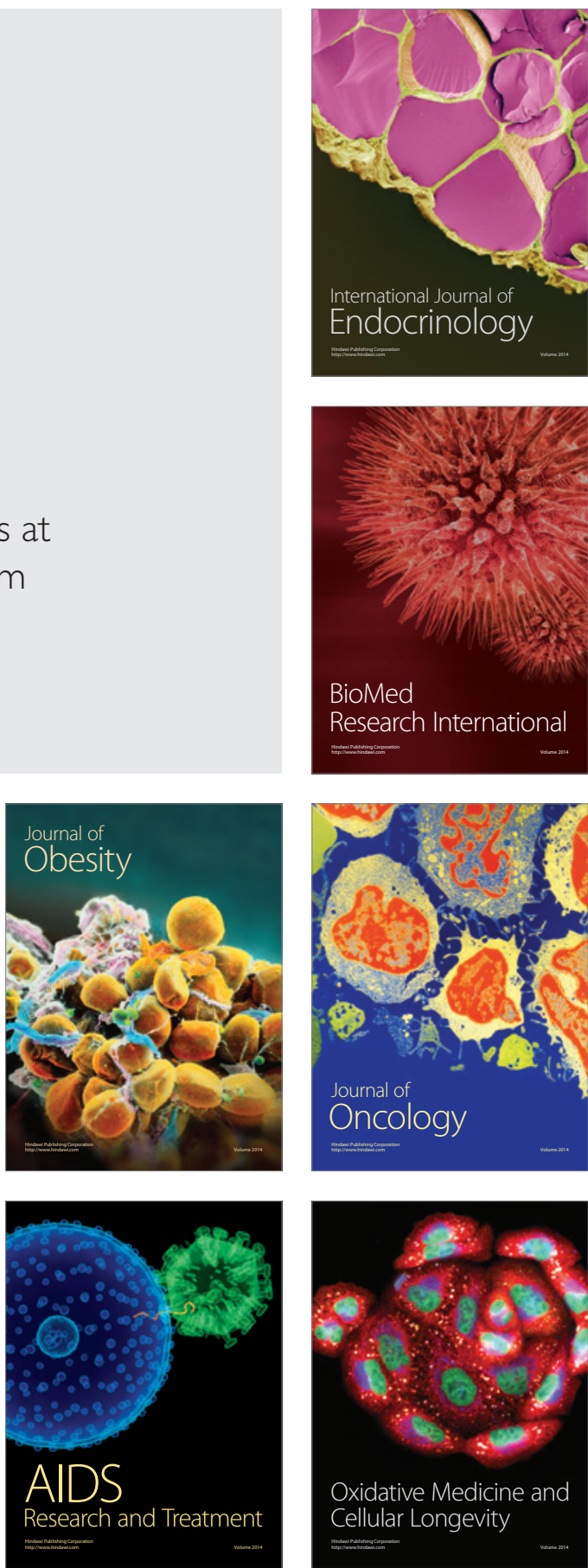